\title{
Three-dimensional graphoepitaxial growth of oxide films by pulsed laser deposition
}

Mozhaev, Peter B.; Mozhaeva, Julia E.; Khoryushin, Alexey V.; Hansen, Jørn Bindslev; Jacobsen, Claus S.; Bdikin, Igor K.; Kotelyanskii, losif M.; Luzanov, Valery A.

Published in:

Physical Review Materials

Link to article, DOI:

10.1103/PhysRevMaterials.2.103401

Publication date:

2018

Document Version

Peer reviewed version

Link back to DTU Orbit

Citation (APA):

Mozhaev, P. B., Mozhaeva, J. E., Khoryushin, A. V., Hansen, J. B., Jacobsen, C. S., Bdikin, I. K., Kotelyanskii, I. M., \& Luzanov, V. A. (2018). Three-dimensional graphoepitaxial growth of oxide films by pulsed laser deposition. Physical Review Materials, 2(10), [103401]. https://doi.org/10.1103/PhysRevMaterials.2.103401

\section{General rights}

Copyright and moral rights for the publications made accessible in the public portal are retained by the authors and/or other copyright owners and it is a condition of accessing publications that users recognise and abide by the legal requirements associated with these rights.

- Users may download and print one copy of any publication from the public portal for the purpose of private study or research.

- You may not further distribute the material or use it for any profit-making activity or commercial gain

- You may freely distribute the URL identifying the publication in the public portal 


$$
4
$$

$$
45
$$

\begin{abstract}
Metaloxide thin films were deposited on tilted-axes $\mathrm{NdGaO}_{3}$ substrates (TAS NGO) by pulsed laser deposition. A specific growth mode resulting in an inclination between crystallographic planes of the top layer and that of the bottom layer was commonly observed. A simple geometrical growth model, taking into account faceting of the surface of the bottom layer, explains the observed dependencies well. The matching of the top and the bottom layer is essentially three dimensional, with graphoepitaxial matching in the substrate plane. The three-dimensional graphoepitaxial (3DGE) growth mechanism seems to be quite common for deposition on TAS with tilt angles more than $5^{\circ}$. It was observed for eight of ten studied combinations of materials, angle compared to the tilt angle of the bottom layer. Two different 3DGE dependencies can be distinguished in the high-angle range $\left(>15^{\circ}\right)$ : with a tendency towards standard growth above some threshold angle, and retaining $3 D G E$ behavior until a tilt angle of $45^{\circ}$ is reached, either by the top or by the bottom layer. In a simplified way the growing grain overgrows another grain, seeded on the next step on the surface, while for the second mechanism the inclination is formed when the grain is seeded over the step. The first type is better by a tangent angular dependence, it is observed usually when a compressive strain is induced in the
\end{abstract} DOI: 10.1103/PhysRevMaterials.00.003400$$
\begin{aligned}
& 45 \text { such a change is the surface structure of the TAS, representing } \\
& 46 \text { a network of steps with terrace and edge surfaces oriented }
\end{aligned}
$$$$
47 \text { along the SICPs (see, e.g., [5]). The SICPs forming the }
$$$$
48 \text { terraces for some substrate materials and orientations are }
$$

\footnotetext{
*Corresponding author: pbmozh@gmail.com
}

\section{INTRODUCTION}

Deposition of epitaxial thin oxide films on substrates with inclination of surface orientation from the small-index crystallographic planes (SICPs) - the tilted-axes substrates (TAS), often also called "miscut" or "vicinal" substrates-is attracting more and more attention in the last decades as a simple way of preparation of quantum wires and quantum dots (see, e.g., [1]). Previously studies of oxide films deposition on TAS was usually pursuing the goal of improvement of the lattice perfection and surface smoothness due to change of the growth mechanism from 3D (Vollmer-Weber or Stransky-Krastanov modes) to the two-dimensional (2D) step-flow growth (see, for example, [2-4]). The reason for 48 terraces for some substrate materials and orientations are

called "habit planes," because the standard deposition on these 49 materials is done with substrate surface orientation along 50 the "habit" SICP. This network of steps provides excellent 51 seeding positions in the internal corners formed by the step 52 edge and the surface of the next terrace, and force the uni- 53 directional growth from the edge of the terrace [Fig. 1(a)]. 54 The overgrowth of the grain seeded on the next joint of 55 terrace and edge determines the general properties of the 56 growing film: strain, orientation, dislocations density, and 57 surface roughness. Note that the strain in the overgrowth area 58 is generated not only by the in-plane mismatch due to the 59 difference of the lattice constants of the film and the substrate, 60 but also by the out-of-plane mismatch [not shown in Fig. 1(a)]. 61 This "standard" growth mode [Fig. 1(a)] demonstrates good 62 parallelism of crystallographic planes of the film and the 63 substrate, and the strain, generated by the film-substrate lattice 64 mismatch, is accommodated by generation of dislocations and 65 step bunching (see, e.g., modeling in [6]). Some authors even 66 claim this standard growth mechanism with parallel SICPs of 67 the film and the substrate to be the only possibility. 


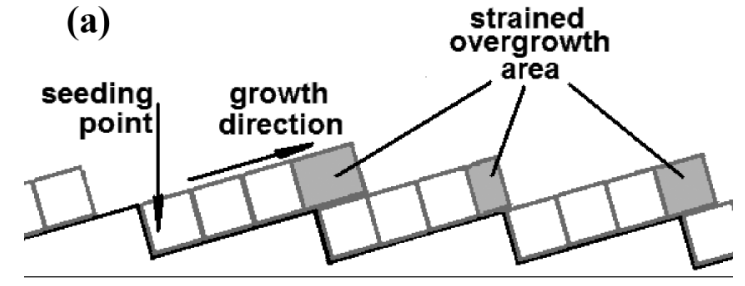

(b)

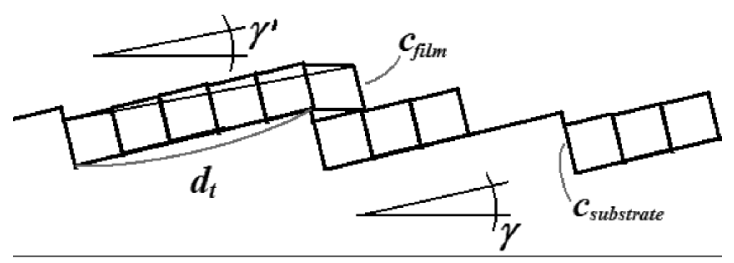

(c)

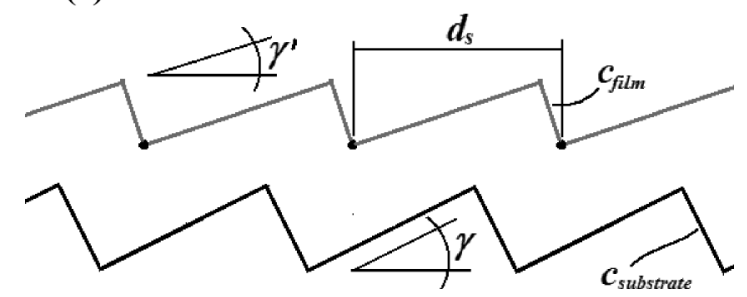

FIG. 1. Growth modes during oxide deposition on tilted-axes substrates: (a) Standard growth mode with parallel small-index crystallographic planes (SICPs) of film and substrate. (b) and (c) Threedimensional graphoepitaxial growth mode with mutual inclination of film and substrate planes. (b) Overgrowth mechanism, when inclination between the SICPs of the substrate and the film is caused by a difference between the height of the substrate step and the layer thickness of the film on the next terrace. The case of smaller height of the growth step of the film is shown. The resulting tilt follows the tangent formula [Eq. (1) in text]. (c) Simultaneous seeding of the film on neighboring seeding knots (black dots) results in the sine model [Eq. (2)].

In fact, studies of deposition of semiconducting thin films 70 on TAS showed the possibility of different film-substrate ori71 entational relations, depending on the mechanism of lattice72 mismatch strain accommodation. The first observations of 73 inclination of the SICPs of the film from that of the substrate 74 date back to the early 1970s [7-10]. The mechanisms resulting 75 in such inclinations were discussed in different ways in [1176 13]. According to [13], three epitaxial modes are possible, 77 with lattice mismatch accommodation by (i) dislocations 78 with Burgers vector in the habit plane, (ii) dislocations with 79 Burgers vector inclined relative to the habit plane, and (iii) so disconnections generated on the edges of the terraces on the 1 TAS surface. The first mode corresponds to the standard mode 32 [Fig. 1(a)], with no inclination of the SICPs of the film from ${ }_{3}$ that of the substrate, and with the lattice-mismatch generated 84 strain being completely relaxed by generation of dislocations. ${ }_{85}$ The second mode results in some (usually small) inclination 86 of the lattice of the crystallites of the growing film, it is ${ }_{87}$ usually detected as an increased width of the out-of-substrate plane rocking curve of the film compared to the corresponding 88 rocking curve of the substrate. The inclination mainly depends 89 on the mismatch between the substrate and film, and for 90 deposition on a substrate aligned along a SICP (habit plane) 91 the crystallites are inclined randomly, or almost randomly, in 92 the substrate plane. Systematic small-angle (usually below $1^{\circ}$ ) 93 tilt of the films SICP along some crystallographic direction 94 in the substrate plane also manifests this second relaxation 95 mechanism (see, e.g., [14]). The third epitaxial mode is a re- 96 sult of accommodation of strain by disconnections generated 97 on the edges of the steps. The inclination of the film lattice 98 depends on both lattice mismatch and substrate plane tilt from 99 the habit plane and may be described in a simple geometric 100 way first proposed by Nagai [8]. The height of the growth 101 steps of the film $c_{f}$ [Fig. 1(b)] is not equal to the height of ${ }_{102}$ the steps on the substrate surface $c_{s}$. As a consequence, the 103 inclination of the crystallographic planes of the film from the 104 substrate surface plane $\gamma^{\prime}$ increases compared to the substrate 105 tilt $\gamma$ when $c_{f}>c_{s}$, and decreases when $c_{f}<c_{s}$. Considering 106 overgrowth of the layers (see, e.g., [15]) with average length 107 of the terrace surfaces $d_{t}$ [Fig. 1(b)], we can write the obvious 108 relations

$$
\begin{aligned}
d_{t} & =c_{f} / \tan \gamma^{\prime}=c_{s} / \tan \gamma, \\
\gamma^{\prime} & =\arctan \left[\left(c_{f} / c_{s}\right) \tan \gamma\right] .
\end{aligned}
$$

This simple formula may change if seeding is considered 110 not in a single edge-terrace joint, but simultaneously on 111 neighboring seeding knots (black dots in Fig. 1(c), [16]). In 112 this case simple considerations provide the sine dependence ${ }_{113}$ instead of tangent:

$$
\begin{aligned}
& d_{s}=c_{f} / \sin \gamma^{\prime}=c_{s} / \sin \gamma, \\
& \gamma^{\prime}=\arcsin \left[\left(c_{f} / c_{s}\right) \sin \gamma\right],
\end{aligned}
$$

where $d_{s}$ is the average distance between the seeding knots on 115 the substrate surface. Usually the authors do not distinguish 116 the two possible mechanisms and use sine or tangent for their ${ }_{117}$ convenience, or even ignore the trigonometric functions and ${ }_{118}$ calculate the angle directly. The reason is the vicinal range of ${ }_{119}$ the tilt angles, in most of the studies less than $5^{\circ}$ and only in 120 some studies increasing to $\sim 10^{\circ}$.

It is important to note that $c_{f}$ and $c_{s}$ are not the lattice ${ }_{122}$ constants, but the heights of the steps of film and substrate, ${ }_{123}$ only in some cases being equal to the lattice constants of the ${ }^{124}$ materials in respective directions (normal to the correspond- ${ }_{125}$ ing habit plane). For example, the height of step can be $1 / 2$ or 126 $1 / 3$ of the lattice constant ( [17] and [15], correspondingly), or ${ }_{127}$ a fractional part of the translation distance in the cases when ${ }_{128}$ faceting happens along (110) or (111) SICPs.

This epitaxial growth mode is essentially three dimen- ${ }_{130}$ sional: the tilt axis of the substrate provides initial bonding ${ }_{131}$ conditions for the film, usually similar to the epitaxial rela- ${ }_{132}$ tions on the habit plane, while the translation distance $\left(d_{t}\right.$ or ${ }_{133}$ $d_{s}$ ) and the ratio of substrate to film step heights determines ${ }_{134}$ the mutual orientation of the habit plane of the substrate and ${ }_{135}$ the corresponding SICP of the film. The film and the substrate ${ }_{136}$ are coupled, thus, in all three dimensions, while for ordinary ${ }_{137}$ epitaxial growth the coupling occurs only in the substrate ${ }_{138}$ plane, i.e., in two dimensions. At the same time, this growth 139 mechanism may be considered as a kind of graphoepitaxy, 140 
141 because the orientation and structure of the film is determined

142 not exclusively by ions in the lattices of film and substrate,

143 but also by a net of features on the substrate surface with the

144 size greater than the interatomic distances or lattice constants.

145 To indicate all these features we will refer to this growth

146 mechanism as the three-dimensional graphoepitaxial (3DGE)

147 growth mode.

${ }_{148}$ The three mechanisms of mismatch relaxation may co149 incide, with corresponding change of inclination angle and 150 strain to some intermediate values between the pure cases 151 [13].

152 The 3DGE mechanism, fairly described with geometrical 153 approximation, was observed in numerous studies of semi154 conductor heteroepitaxy, references can be found in [11155 13]. Most of these heterostructures were limited to small tilt 156 angles (vicinal range), utilized in semiconductor technology

157 for improvement of thin film quality. Oxide thin films and 158 substrates were rarely studied and most of the obtained tilts 159 in oxide heterostructures were misinterpreted or left without 60 explanation.

161 To our knowledge, 3DGE in all-oxide heterostructures was 162 first observed in 1991 by Kotelyanskii and Luzanov [18], 163 when $\mathrm{CeO}_{2}$ films were deposited on $\mathrm{NdGaO}_{3}$ (NGO) TAS 164 (tilt around the [001] axis from the (110) plane towards 165 (010) plane) with e-beam evaporation. All range from (110) 166 to $\left(\begin{array}{lll}-1 & 1 & 0\end{array}\right)$ planes [orthorhombic notation, equivalent to the $167(100)_{c}$ and $(010)_{c}$ planes of the pseudocubic notation for 168 the NGO crystal] was studied. The $\langle 110\rangle$ axis of the $\mathrm{CeO}_{2}$ 169 film was bound to the [001] tilt axis of the substrate for all 70 TAS orientations. The inclination of the $\mathrm{CeO}_{2}$ (001) SICP 171 from the sample surface monotonously increased surpassing 172 the increase of the substrate tilt angle, until (110) $\mathrm{CeO}_{2}$ 173 orientation was reached at $\gamma_{c} \approx 32^{\circ}$ [Fig. 2(a), solid line]. 174 The faster increase of film tilt $\gamma^{\prime}$ compared to the substrate 175 tilt $\gamma$ was due to greater lattice constant of the film (5.4 176 and $3.86 \AA$, correspondingly), and showed good agreement 177 with the simple formula (2). The film remained (110) ori178 ented with a wide spread of grains orientation (rocking curve 179 width $\sim 5^{\circ}$ ) until $\sim 58^{\circ}$ substrate tilt, when 3DGE started to 180 follow the $\left(\begin{array}{lll}-1 & 1 & 0\end{array}\right)$ crystallographic plane of the substrate, 181 symmetrically equivalent to the initial (110) NGO plane. 182 The reason for (110)-oriented film growth is the presence of 183 two symmetrically equivalent $\{110\}$ SICPs on the substrate 184 surface, each of them showing no preference over the other 185 neither in the resulting tilt of the film, nor in the area of the 186 corresponding facets on the substrate surface. The critical tilt 187 angle at which the growth mode changes from 3DGE to (110) 188 oriented is given by simple formula [sine dependence of tilt 189 (2)]

$$
\gamma_{c}=\arcsin \left[\left(c_{s} / c_{f}\right) \sin \left(45^{\circ}\right)\right]
$$

For $\mathrm{CeO}_{2}$ deposition on $\mathrm{NGO} \gamma_{c} \approx 30.4^{\circ}$, in a reasonable agreement with the observed value.

The study of $\mathrm{CeO}_{2} / \mathrm{NGO}$ heterostructures was continued using rf sputtering $[19,20]$ and pulsed laser deposition (PLD) techniques $[16,21]$. Much higher deposition rate during $\mathrm{rf}$ sputtering $(5-7 \mathrm{~nm} / \mathrm{min}$ instead of $0.5 \mathrm{~nm} / \mathrm{min}$ for e-beam evaporation) resulted in a more complicated behavior $[19,20]$. Both standard growth mode (type I in $[19,20]$ ) and 3DGE
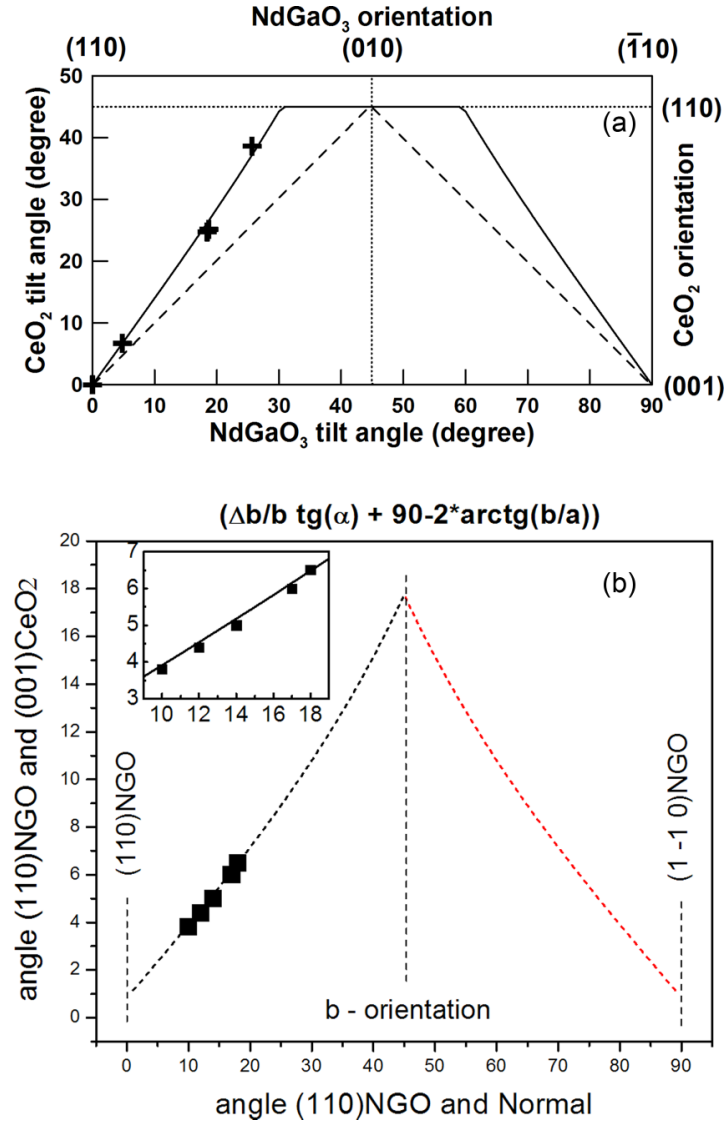

FIG. 2. First observations of gradual increase in tilt of crystallographic planes of the film in $\mathrm{CeO}_{2}$ deposition on $\mathrm{NdGaO}_{3}$ tilted-axes substrates. (a) With e-beam evaporation (solid line, [18]) and rf sputtering (crosses, $[19,20]$ ). The tilt of $\mathrm{CeO}_{2}$ film with standard epitaxial growth mode is shown by a dashed line. (b) With PLD [22]. The formula on the graph takes into account the orthorhombicity of $\mathrm{NdGaO}_{3}$. Note that dependencies follow the sine formula [Eq. (1)] for (a) and the tangent formula [Eq. (2)] for (b).

growth mode (type II) are degenerated twice due to the pres- 199 ence of symmetrically equivalent $(110)$ and $\left(\begin{array}{lll}-1 & 1 & 0\end{array}\right)$ planes 200 on the substrate surface. The angular dependence of the 3DGE 201 part of the film followed the same sine mode (2) as for 202 e-beam evaporation [Fig. 2(a), crosses]. Further increase of 203 the deposition rate for PLD (7-55 nm/min average deposition 204 rate, depending on the laser repetition rate, and above 100205 $\mathrm{nm} / \mathrm{min}$ peak deposition rate during the laser pulse) provided 206 $3 \mathrm{DGE}$ growth in the $4^{\circ}-20^{\circ}$ substrate tilt angle range [21]. At 207 higher angles the inclination between the NGO (110) plane 208 and the $\mathrm{CeO}_{2}(001)$ plane dropped to $2^{\circ}-3^{\circ}$ and remained at 209 this level over the range $\left(23^{\circ}<\gamma<30^{\circ}\right)$. Formula (1) was 210 applied to the 3DGE growth during PLD in [16] [Fig. 2(b)] 211 and showed excellent agreement with the observed mutual 212 film and substrate orientation. The more complicated view 213 of the formula in Fig. 2(b) compared to formula (1) is due 214 to an attempt to take into account the orthorhombic structure 215 of the substrate; in fact, this correction is small and may be 216 neglected. Deposition of $\mathrm{YBa}_{2} \mathrm{Cu}_{3} \mathrm{O}_{x}$ (YBCO) over the $\mathrm{CeO}_{2}{ }_{217}$ layer on NGO TAS resulted in a similar linear increase of the 218 
19 film inclination and abrupt drop to $\sim 2.5^{\circ}$ when $\gamma$ exceeded $25^{\circ}[21]$.

221 PLD of $\mathrm{CeO}_{2}$ buffer layer on Ni biaxially textured tape 222 with grain orientations randomly spread from the substrate 223 surface plane was studied in [17]. At high deposition tem224 peratures the out-of-substrate plane tilt of the (001) plane 225 of the $\mathrm{CeO}_{2}$ overlayer follows the tilt of the (001) plane of 226 the $\mathrm{Ni}$ grain below, but is smaller, in a qualitative agreement 227 with the geometric formula. At low deposition temperature 228 the crystallites of $\mathrm{CeO}_{2}$ are smaller than the terrace width 229 on the $\mathrm{Ni}$ grain surface; this excludes the graphoepitaxial 230 effect of steps on the film orientation, and, in fact, the $\mathrm{CeO}_{2}$ 231 film grows in an agreement with the standard growth mode, ${ }_{232}(001) \mathrm{CeO}_{2} \|(001) \mathrm{Ni}$ [17]. Authors mention that grains of ${ }_{23} \mathrm{LaMnO}_{3}$ and Y-stabilized $\mathrm{ZrO}_{2}$ (YSZ) also tilted according 234 to the geometrical model when deposited on Ni tapes [17].

235 Similarly, in [15] another fluorite material, YSZ, deposited 236 on the vicinal sapphire substrate $\left[5^{\circ}\right.$ from (0001) plane] with 237 liquid phase epitaxy (LPE), showed mixed orientation from 238 the standard growth mode to the 3DGE growth mode. The 239 reason was intense step bunching on the substrate surface dur240 ing substrate preparation (annealing at $1500{ }^{\circ} \mathrm{C}$ ), resulting in 241 broad (0001)-oriented terraces. Some of the YSZ crystallites 242 were small enough to fit one terrace, and showed orientation 243 (001) YSZ $\mid(0001) \mathrm{Al}_{2} \mathrm{O}_{3}$, while orientation of the big YSZ 244 grains showed a tilt of $5.9^{\circ}$, in an excellent agreement with 245 the geometrical model [15].

246 YBCO deposition by PLD on YSZ TAS and $\mathrm{CeO}_{2}$-buffered 247 sapphire TAS was demonstrated in [22]. Both cases showed 248 DDGE, assuming that the $\mathrm{CeO}_{2}$ buffer layer is well oriented 249 along the sapphire SICP : $(001) \mathrm{CeO}_{2} \|\left(\begin{array}{llll}1 & -1 & 0 & 2\end{array}\right) \mathrm{Al}_{2} \mathrm{O}_{3}$. 250 We will discuss the results of [22] in more detail in the 251 Discussion section below.

252 YBCO deposition on YSZ TAS was also studied in [23].

${ }_{253}$ Direct deposition resulted in $c$-oriented YBCO films inde254 pendent of the substrate tilt angle, but introduction of a 255 buffer $\mathrm{Y}_{2} \mathrm{O}_{3}$ layer between $\mathrm{YBCO}$ and $\mathrm{YSZ}$ blocked chemical 256 interaction and promoted film growth with a tilt of SICP. The 257 inclination of the YBCO film from the habit plane shows the ss 3DGE behavior (increase of the inclination with an increase 259 of the substrate tilt angle), but the measured value of the film 260 tilt is less than calculated using the simple formula (1). At 261 high substrate tilt angle $\left(35.7^{\circ}\right)$ the YBCO film grows in the 262 standard epitaxial mode with small (less than $1^{\circ}$ ) deviation 263 from the habit plane (001) YSZ [23].

$264 \mathrm{PbTiO}_{3}$ deposition on $\mathrm{SrTiO}_{3}$ TAS [24] showed good ${ }_{265}$ agreement with the geometrical model, even though the mech266 anism of growth is much more complicated than in other dis267 cussed studies ( $a$-oriented grains formation on the edges of the 268 steps, with corresponding strains and distortions introduced 269 into the $c$-oriented grains on the terraces). Another study of $270 \mathrm{PbTiO}_{3}$ deposition on TAS, with $\mathrm{MgO}$ substrates [25], also 271 resulted in the growth similar to the 3DGE, but with higher 272 inclination of the film compared to the calculated using the 273 table data.

274 Summarizing, the 3DGE growth mode is not something 275 unusual for all-oxide heterostructures on TAS, but the mech276 anism was quite often not recognized, and the results were 277 misinterpreted. Systematic investigations of the growth mode 278 are lacking, the angular ranges of 3DGE growth were not determined. Deviations from the simple geometrical formula 279 were not explained. At the same time, understanding of the 280 3DGE mechanism is important for the fabrication of thin 281 film heterostructures, especially when the materials are highly 282 anisotropic, like high-temperature superconductors (HTSC) 283 or piezoelectrics.

In this paper we present our observations of the 3DGE 285 growth in different film-substrate pairs. Preservation of the 286 3DGE growth mode through a multilayer system is demon- ${ }^{287}$ strated, and the characteristic features of the growth mode and 288 deviations from the simple geometrical model are discussed. 289 In this study we will concentrate on orientational characteris- 290 tics of the 3DGE films and multilayers, the fabrication detail 291 and secondary growth features will be presented in another 292 publication.

\section{EXPERIMENTAL TECHNIQUES}

294

The TAS $\left(5 \times 5 \times 0.5 \mathrm{~mm}^{3}\right)$ were cut from $\mathrm{NdGaO}_{3}$ sin- 295 gle crystals, their substrate surface was set by tilting from 296 the (110) habit plane around the [001] axis towards the 297 (010) plane (corresponding to tilt around the $[001]_{\mathbf{c}}$ axis 298 from the $(100)_{c}$ plane towards the $\left(\begin{array}{lll}-1 & 1 & 0\end{array}\right)_{c}$ plane in the ${ }^{299}$ pseudocubic notation). The nominal tilt angle varied in the 300 range $0^{\circ}-34^{\circ}$. Chemical-mechanical polishing (CMP) of the 301 substrates provided atomically flat surfaces with a roughness 302 $R_{a}$ determined by atomic force microscopy (AFM) below 303 $2 \AA$ A. Such a low roughness implies presence of a damaged 304 "amorphous" layer on the surface of the substrate after CMP. ${ }_{305}$ Formation of pronounced steps on the surface with terraces 306 and edges oriented along the $\{110\}$ planes (surface recrystal- 307 lization) demanded additional treatment: wet etching with HF 308 and high-temperature annealing in oxygen (to be published). 309 Mainly depositions were done on the as-polished substrates, 310 only after rigorous cleaning in organic solvents and weak acid 311 to remove contaminants present after dicing and CMP. The 312 actual tilt orientation and angle of the substrate surface were ${ }_{313}$ checked after CMP with XRD measurements. The deviation ${ }^{314}$ of the actual tilt axis from the [001] axis of $\mathrm{NdGaO}_{3}$ did not ${ }_{315}$ exceed $5^{\circ}$, being usually less than $2^{\circ}$. The actual tilt angle was 316 measured for each substrate.

Fluorite $\mathrm{YSZ}$ and $\mathrm{CeO}_{2}$, perovskite $\mathrm{BaZrO}_{3}(\mathrm{BZO})$, and ${ }_{318}$ perovskitelike YBCO thin films were deposited in differ- 319 ent combinations with PLD. The details of the technique 320 can be found in [26]. Commercially available stoichiometric ${ }_{321}$ high-density ( $>90 \%$ of bulk density) ceramic targets were ${ }_{322}$ used. The structural properties of thin films and multilayers 323 were studied using x-ray diffraction techniques, the surface ${ }^{324}$ morphology was observed by SEM and AFM. Electrical 325 properties of the superconducting films were measured with 326 noncontact techniques. The complete results of our studies, ${ }_{327}$ including morphology and electrical properties of the films, 328 will be published elsewhere: in this article we will concentrate ${ }_{329}$ only on the orientational features of the heterostructures. 330

The chosen film and substrate materials provided a wide 331 range of lattice mismatch and corresponding strain introduced ${ }_{332}$ into the upper layer. The translation distances at room tem- 333 perature and expected strain in the habit plane (110) NGO ${ }_{334}$ are presented in Table I for all studied top layer/bottom layer 335 combinations. The lattice mismatches, in fact, differ from the 336 
TABLE I. Lattice parameters of the substrate and film materials.

\begin{tabular}{|c|c|c|c|c|c|c|}
\hline \multirow{3}{*}{$\begin{array}{l}\text { Top layer } \\
\text { Bottom layer }\end{array}$} & & & \multicolumn{4}{|c|}{ Calculated in-plane strain $^{\mathrm{a}}(\%)$} \\
\hline & \multicolumn{2}{|c|}{ Translation distances $^{\mathrm{b}}(\AA)$} & $\mathrm{Y}: \mathrm{ZrO}_{2}$ & $\mathrm{CeO}_{2}$ & $\mathrm{BaZrO}_{3}$ & $\mathrm{YBa}_{2} \mathrm{Cu}_{3} \mathrm{O}_{x}$ \\
\hline & in-plane & out-of-plane & & & & \\
\hline $\begin{array}{l}\mathrm{NdGaO}_{3} \\
\text { Orthorhombic }\end{array}$ & $3.861 \times 3.864^{\mathrm{c}}$ & 3.861 & $+5.7-+5.8$ & $+1.0-+1.1$ & $-8.7--8.8$ & $-0.75-+1.0$ \\
\hline $\begin{array}{l}Y: \mathrm{ZrO}_{2} \\
\text { Cubic }\end{array}$ & $3.641^{\mathrm{d}}$ & 5.149 & $\mathrm{xxx}$ & -5.0 & -15.4 & $-6.8--5.1$ \\
\hline $\begin{array}{l}\mathrm{CeO}_{2} \\
\text { Cubic }\end{array}$ & $3.822^{\mathrm{d}}$ & 5.405 & & $\mathrm{xxx}$ & & $-1.8--0.1$ \\
\hline $\begin{array}{l}\mathrm{BaZrO}_{3} \\
\text { Cubic }\end{array}$ & 4.20 & 4.20 & & & $\mathrm{xxx}$ & $+7.4-+8.9$ \\
\hline $\begin{array}{l}\mathrm{YBa}_{2} \mathrm{Cu}_{3} \mathrm{O}_{x} \\
\text { Orthorhombic }\end{array}$ & $3.825 \times 3.89$ & $3.89-3.92^{\mathrm{e}}$ & & & & $\mathrm{xxx}$ \\
\hline
\end{tabular}

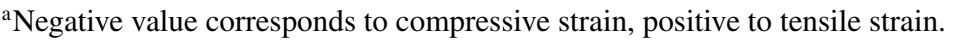

b"In-plane" data are provided for the standard (110) orientation of the NGO substrate and corresponding SICPs of the films. Lattice structure and parameters are given for room temperature.

${ }^{c}$ Orthorhombic lattice of $\mathrm{NdGaO}_{3}$ results in orthogonal translation directions on the (110) plane with distances $c / 2=3.864 \AA$ and $\left[\left(a^{2}+b^{2}\right)^{1 / 2}\right] / 2=3.861 \AA$.

${ }^{\mathrm{d}}$ Fluorite lattice during deposition on perovskite $\mathrm{NdGaO}_{3}$ substrate is tilted in substrate plane by $45^{\circ}$ and the in-plane translation distances corresponding to the substrate axes are calculated as $5.149 / 2^{1 / 2}=3.641$ and $5.405 / 2^{1 / 2}=3.822 \AA$ for $\mathrm{YSZ}_{\text {and }} \mathrm{CeO}_{2}$, respectively.

${ }^{\mathrm{e}}$ Perovskitelike lattice of YBCO consists of three perovskite cells in $c$ direction, so the out-of-plane translation distance is given by $c / 3=$ 3.89-3.92 A, depending on the oxygen contents in the film.
}

337 presented, as a result of different thermal expansion coeffi338 cients, but since the lattices of both layers can be distorted by 339 the substrate-induced strain, by presence of oxygen vacancies, 340 and by chemical interaction between layers, we cannot present 341 exact values of the strain and keep the room-temperature data 342 as a reference. Actual strain should be determined for each 343 top layer/bottom layer combination using the measured lattice 344 constants in the fabricated heterostructures.

345 The deposition parameters for $\mathrm{CeO}_{2}$ and YSZ were op346 timized to obtain smooth thin films of single orientation $347(001) \mathrm{CeO}_{2} \|(110) \mathrm{NGO}$ on a standard (110) NGO substrate. 348 Substrate temperature during deposition was held at $740{ }^{\circ} \mathrm{C}$, 349 the target was ablated at an energy density of $1.1 \mathrm{~J} / \mathrm{cm}^{2}$ and 350 pulse repetition rate of $2 \mathrm{~Hz}$ in a mixture of argon and oxygen $351\left(6 \% \mathrm{O}_{2}, 0.2 \mathrm{mbar}\right.$ total pressure). Low energy density just 352 above the ablation threshold $\left(\sim 1 \mathrm{~J} / \mathrm{cm}^{2}\right.$ in our deposition 53 system) resulted in a very low growth rate $(\sim 0.3 \AA /$ pulse $=$ $3540.6 \AA / \mathrm{s}$ for $\mathrm{CeO}_{2}$ and $\sim 0.1 \AA /$ pulse $=0.2 \AA / \mathrm{s}$ for $\mathrm{YSZ}$ ), and low oxygen partial pressure promoted growth of a film 355 of high crystal quality. No post-deposition annealing was 356 performed; the film was cooled down to room tempera- 357 ture in the working atmosphere at the maximal possible 358 rate.

The lattice constant of the YSZ films on (110) NGO ${ }_{360}$ substrates was typically 5.145-5.155 , for some samples 361 ranging from 5.135 to $5.185 \AA$, in good agreement with 362 standard value of $5.15 \AA$. The FWHM (full width at half ${ }_{363}$ maxima) of the peaks on the $\theta / 2 \theta$ scans for the YSZ films 364 was very close to the estimations of the size broadening, 365 implying high homogeneity and low strain in the films. The 366 $\mathrm{CeO}_{2}$ films on (110) NGO substrates showed lattice constant 367 5.395-5.41 $\AA$, close to the standard 5.4 $\AA$. The FWHM of the 368 peaks on the $\theta / 2 \theta$ scans was significantly higher than the 369 size broadening, indicating strained or inhomogeneous layer 370 formation. Both YSZ and $\mathrm{CeO}_{2}$ films showed wide rocking 371 curves (see Table II).

TABLE II. Typical structural properties of the films grown in standard epitaxial mode on low-angle TAS NGO. The spread of the presented parameters is $\sim 50 \%$. Actual film properties strongly depend on deposition conditions.

\begin{tabular}{|c|c|c|c|c|c|c|}
\hline Film/substrate & $\begin{array}{c}\text { Lattice } \\
\text { mismatch }^{\mathrm{a}}(\%)\end{array}$ & $\begin{array}{c}\text { Lattice } \\
\operatorname{constant}^{\mathrm{b}}(\AA)\end{array}$ & $\begin{array}{c}\text { Strain } \Delta d / d \\
(\%)\end{array}$ & $\begin{array}{l}\text { FWHM of rocking } \\
\text { curve (deg) }\end{array}$ & $\begin{array}{c}\text { Film SICP } \\
\text { inclination }(\mathrm{deg})\end{array}$ & $\begin{array}{c}\text { Vicinal range } \\
\text { (deg) }\end{array}$ \\
\hline YSZ/NGO & $+5.7-+5.8$ & 5.149 & 0.25 & 1.1 & $<0.5$ & 2 \\
\hline $\mathrm{CeO}_{2} / \mathrm{NGO}$ & $+1.0-+1.1$ & 5.405 & 0.15 & 0.5 & $<0.15$ & 1 \\
\hline $\mathrm{YBa}_{2} \mathrm{Cu}_{3} \mathrm{O}_{x} / \mathrm{CeO}_{2}$ & $-0.75-+1.0$ & 11.685 & 0.4 & 0.35 & $<0.1$ & 1 \\
\hline $\mathrm{BaZrO}_{3} / \mathrm{NGO}$ & $-8.7--8.8$ & 4.200 & 0.6 & 0.5 & $<0.1$ & no data \\
\hline
\end{tabular}

${ }^{a}$ Negative value corresponds to compressive strain, positive to tensile strain.

${ }^{b}$ Normal to substrate plane. 
373 BZO films were deposited at the same conditions as fluorite 374 films. The deposition rate for BZO was $0.225 \AA /$ pulse $=$ $3750.45 \AA / \mathrm{s}$. The BZO films showed good lattice perfection as 376 determined by XRD $\theta / 2 \theta$ scans and rocking curves (Table II). 377 The lattice constant was 4.197-4.213 $\AA$, in good agreement 378 with the bulk value $(\sim 4.2 \AA)$.

The YBCO thin films deposition parameters (1.2-1.5 J/ $\mathrm{cm}^{2}$, oxygen partial pressure $0.16 \mathrm{mbar}$, total pressure of $\mathrm{Ar} / \mathrm{O}_{2}$ mixture $0.8 \mathrm{mbar}$ ) were optimized to 2 obtain the best superconducting and structural properties for the films grown on the standard (110) NGO substrates. 384 Relatively low deposition temperature of $730-750{ }^{\circ} \mathrm{C}$ 385 suppressed the chemical interaction of $\mathrm{YBCO}$ with $\mathrm{CeO}_{2}$ 386 bottom layer in multilayer structures. The deposition rate of ${ }_{387} 0.8 \AA$ /pulse $(1.6 \AA / \mathrm{s}$ at standard $2 \mathrm{~Hz}$ laser pulse repetition 388 rate) provided enough time for relaxation of the deposited 389 material on the surface of the growing film. A prebake step 390 before deposition saturated the substrate surface with oxygen 391 and decreased the probability of chemical interaction with 392 the growing film. As a consequence, the lattice perfection 393 of the film significantly increased, especially for the thin 394 layer near the interface with the substrate [26], and both 395 size and density of the particles on the thin film surface 396 decreased. Post-deposition annealing was performed at $397450{ }^{\circ} \mathrm{C}$ in 800 mbar of oxygen for $1 \mathrm{~h}$. All YBCO films 398 showed $T_{c}$ above $89 \mathrm{~K}$ and a narrow superconducting 399 transition, proving good uniformity of the film structure. 400 The $c$ lattice constant for all films was $11.67-11.7 \AA$, 401 confirming good reproducibility of the film fabrication 402 procedure. Rocking curve width, FWHM of the peaks of the $403 \theta / 2 \theta$ scans, and strain estimation, depended on tilt angle, 404 underlying material, and deposition conditions, and varied 405 significantly.

406 Multilayers were usually prepared ex situ to have a 407 possibility to study the bottom layer properties before 408 and after deposition of the top layer. Some multilayer 409 structures were fabricated in situ, their parameters were 410 compared with that of corresponding ex situ fabricated 411 heterostructures.

412 Deposition rate of the deposited materials was calibrated 413 using selective wet chemical etching of grown films or with 414 lift-off removal of some part of the fabricated layer using 415 a predeposited and patterned hard mask. The value calcu416 lated using a number of pulses on target and calibrated 417 deposition rate we call the nominal thickness. Actual film 418 thickness may differ from the nominal value not only as a 419 result of small deviations of deposition parameters, but also 420 due to modification of the target surface by laser irradia421 tion, usually resulting in a lower ablation rate with time. 422 The thickness was also evaluated using the Williamson-Hall 423 method, the result was in good agreement with the nominal 424 value (error below 15\%). Application of the Williamson-Hall 425 calculation allowed also determination of the variation of 426 the diffraction period normal to the diffraction plane $\Delta d / d$. ${ }_{427}$ This parameter, usually called "strain," was determined for 428 films of all materials if more than one diffraction peak was 429 present on the $\theta / 2 \theta$ scan. Note that this is not the strain 430 related to the film-substrate lattice mismatch (Table I), though 431 sometimes a relation can be established between these two 432 parameters.

\section{RESULTS}

433

The studies of epitaxial growth in semiconductor het- 434 erostructures showed that the tilt axis acts as an anchor setting 435 the initial epitaxial relation along the habit plane. Similarly, in 436 our previous studies [19-21] the $\langle 100\rangle$ axes of the perovskite ${ }_{437}$ films and the $\langle 110\rangle$ axes of the fluorite films were parallel to 438 the substrate tilt axis [001] NGO for all deposition conditions. 439 In this study we assumed that this epitaxial relation remains 440 correct and limited the XRD studies to $\theta / 2 \theta$ and $\omega$ scans 441 around the substrate tilt axis with an initial offset angle $\omega_{0} . \quad{ }_{442}$ Our assumption is corroborated by similar integral intensity of ${ }_{443}$ the XRD peaks for the films with the same nominal thickness. 444 When the observed peak integral intensity was significantly 445 smaller, we performed a search for additional orientations. All 446 orientations found still followed the same epitaxial relation ${ }_{447}$ with some of the film axes parallel to the substrate tilt axis. $\quad 448$

To avoid misunderstanding we will use the following nota- 449 tions:

(i) the substrate plane is the plane of substrate surface; $\quad 451$

(ii) the tilt angle $\gamma$ is the angle between the SICP of film 452 or substrate (habit plane) and the substrate plane; 453

(iii) the inclination angle is the angle between the SICPs 454 of the substrate and the film;

455

(iv) the misorientation is the spread of orientations of 456 individual grains of the film around the main orientation, 457 usually determined as FWHM of the corresponding rocking 458 curve.

\section{A. Standard epitaxial growth}

460

The epitaxial growth for very small tilt angles (vicinal 461 range) does not differ much from growth on a substrate 462 ideally oriented along the habit plane. In fact, the surface 463 of a substrate exactly oriented along the habit plane usually 464 consists of local areas with very small tilt from the habit 465 plane: only the average orientation corresponds to the SICP ${ }_{466}$ (see, for example, discussion of different effects of roughness 467 in [11]). In our case morphological, structural, and electrical 468 parameters of the films remain the same as for the (110) NGO ${ }_{469}$ substrate until some threshold tilt value; this angle determines 470 the vicinal range of epitaxy. Usually in this range the tilt of 471 the film due to the 3DGE growth hardly can be distinguished 472 from the tilt due to other mechanisms, taking into account 473 the accuracy of the XRD measurement and relatively high 474 misorientation of the film grains after PLD. Morphologically 475 the surface of the film shows no specific directions and the 476 grains are rounded or evenly oriented, if elongated. 477

At the same time, the films obtained in the vicinal range 478 with the "standard" epitaxial growth mode may be considered 479 as a reference for the 3DGE grown films at higher tilt angles. 480 For this reason in this section we present the main parame- 481 ters of all films deposited on TAS of the vicinal range (see 482 Table II).

Generally we note that the parameters of the films grown in $\quad 484$ the vicinal range depend on conformity of the lattice structure 485 and the mismatch strain introduced by the substrate. The 486 fluorite films deposited on the perovskite substrates show ${ }_{487}$ a wider rocking curve and higher deviation of the average 488 orientation from the (110) NGO plane compared with the 489 perovskite films, but much lower variation of the out-of-plane 490 


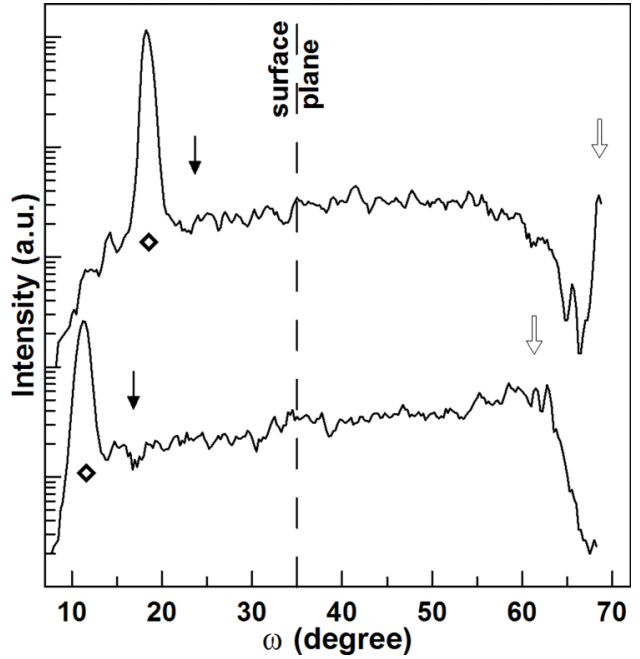

FIG. 3. Rocking curves for the (400) peak of $\mathrm{CeO}_{2}$ films on TAS NGO. Substrate tilt angle: top curve $11.4^{\circ}$, bottom curve $18.4^{\circ}$. Black arrows show the angular position of the (110) NGO plane, white arrows correspond to the angular position of the (010) NGO plane. The 3DGE peaks of $\mathrm{CeO}_{2}$ films are marked with diamonds.

491 lattice constant $\Delta d / d$. Both for fluorite and perovskite films 492 the structural parameters are better for the materials with 493 smaller lattice mismatch.

494 Deposition of YBCO on NGO TAS in all conditions and 495 for all tilt angles resulted in standard epitaxial growth with 496 relations $\langle 100\rangle(001)$ YBCO ||[001](110) NGO. The details of 497 these films structure, morphology, and superconducting prop498 erties can be found in [21,27]. Such preservation of standard 499 growth mode on TAS of all angles is considered as usual for
YBCO deposition on perovskite substrates (see, e.g., [3], and 500 [19-21,27]).

In our previous studies we assumed that a difference in the 502 lattice structure is an important condition of 3DGE growth: 503 a perovskite film on a perovskite substrate (like YBCO on 504 NGO) and a fluorite film on a fluorite underlying layer would 505 follow standard epitaxial growth mode. In fact, this is not true, 506 as we will show below.

\section{B. 3DGE growth \\ 1. $\mathrm{CeO}_{2}$ on NGO TAS}

508

509

Typical rocking curves of $\mathrm{CeO}_{2}$ films deposited by PLD ${ }_{510}$ on TAS NGO are presented in Fig. 3. The 3DGE peak is 511 shifted from the position of the (110) NGO plane towards 512 higher tilt angles, and the shift increases with substrate tilt ${ }_{513}$ angle. All our results for 3DGE growth of $\mathrm{CeO}_{2}$ at different 514 substrate tilt angles are gathered in Fig. 4(a). The film orien- 515 tation dependence on substrate tilt is finely described by the 516 simple geometrical model (1) until $\sim 20^{\circ}$. Above this value 517 the film tilt decreases towards the standard epitaxial relation 518 (001) $\mathrm{CeO}_{2} \|(110) \mathrm{NGO}$. For substrate tilt angles $30^{\circ}-35^{\circ}$ we 519 observed (110)-oriented $\mathrm{CeO}_{2}$ films, with wide rocking curves 520 (up to $4^{\circ}$ ) and high strain $\left(\Delta d / d \approx 1^{\circ}\right.$ ). These results are ${ }_{521}$ in good agreement with [18] and limitations of the simple ${ }_{522}$ geometrical model (3). Detailed description of deposition ${ }_{523}$ technique, (110)-oriented $\mathrm{CeO}_{2}$ films properties, and specific ${ }_{524}$ cases in the angular range $30^{\circ}-45^{\circ}$, will be presented in 525 another publication. The $\mathrm{CeO}_{2}$ film orientation for the tilt ${ }_{526}$ angles $25^{\circ}-30^{\circ}$ remains unclear: we could not reliably detect ${ }_{527}$ weak high-index XRD peaks from the $\mathrm{CeO}_{2}$ film. Formation 528 of a polycrystalline film seems probable, maybe with a set of ${ }_{529}$ predominant orientations.
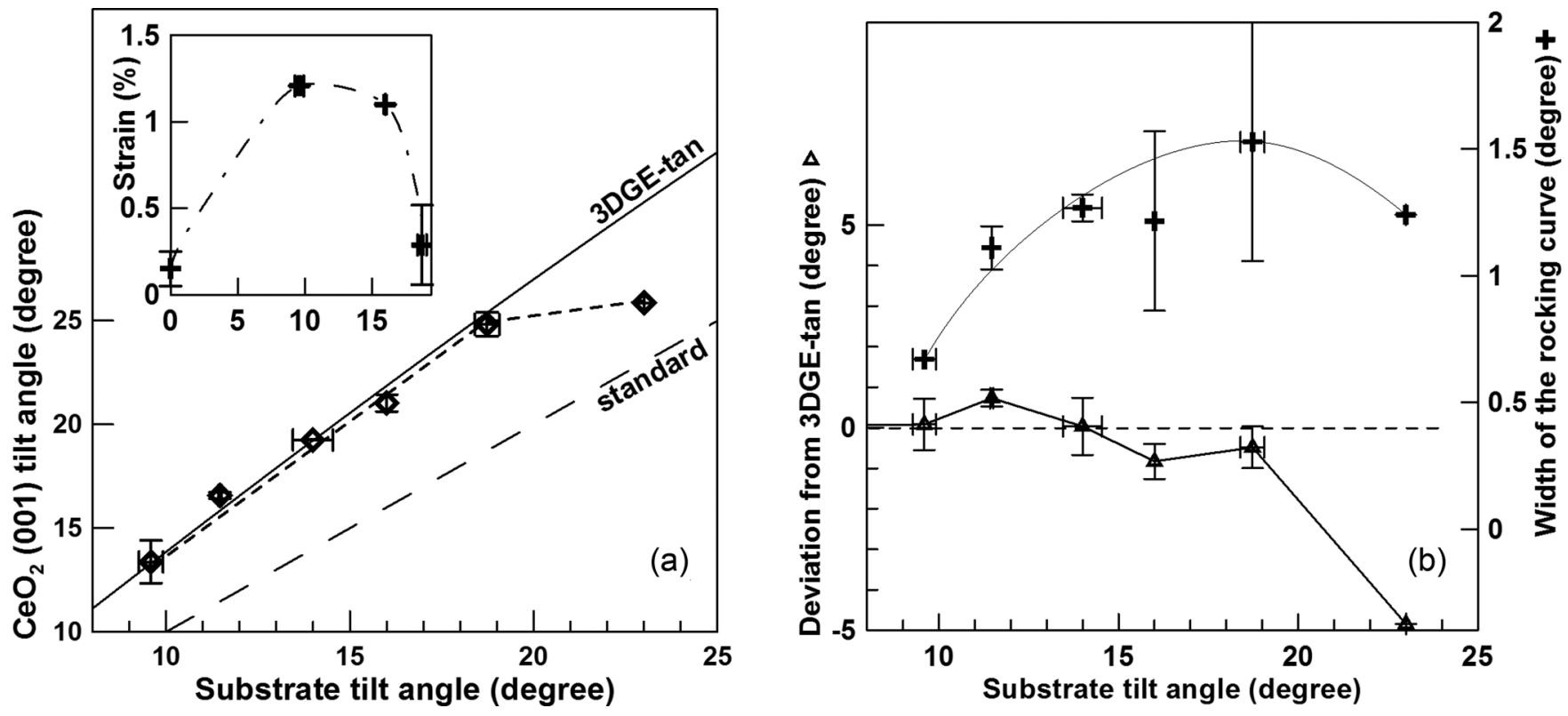

FIG. 4. Orientational relations of $\mathrm{CeO}_{2}$ films on TAS NGO. (a) The film orientation follows the 3DGE-tangent growth mode [Eq. (1)] until $20^{\circ}$, and then the film tilt deviates towards the standard growth mode. Inset: Dependence of the lattice constant variation (strain) on the substrate tilt angle. (b) Deviation from calculated tilt angle (triangles) changes from small positive to small negative with substrate tilt angle until threshold at $20^{\circ}$. The width of rocking curve (crosses) increases with angle. Lines are given as guides for the eye. 


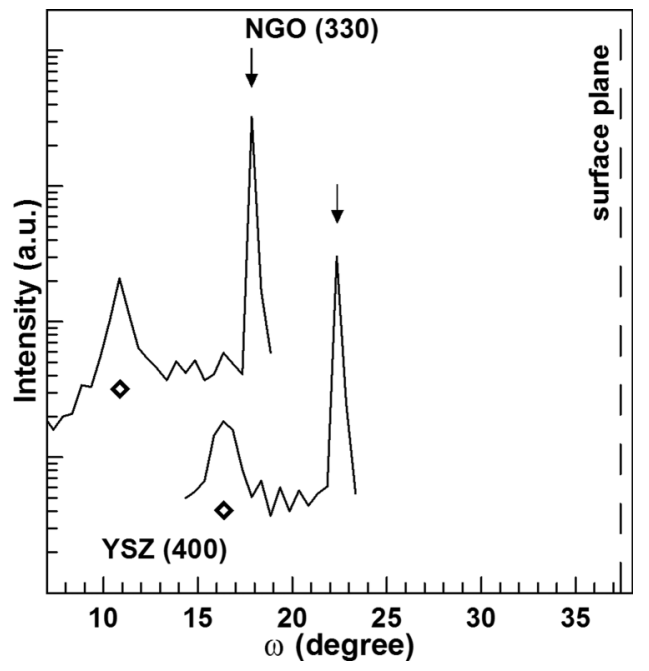

FIG. 5. Rocking curves for the (400) peak of the YSZ films on TAS NGO. Substrate tilt angle: top curve $19.5^{\circ}$, bottom curve $15.4^{\circ}$. Arrows show the angular position of the (110) NGO plane, the 3DGE (400) YSZ peaks are marked with diamonds.

531 The deviation of the film orientation from the calculated 532 value depends on film properties and deposition parameters. 533 On average, the 3DGE film shows tilt angle slightly $\left(<1^{\circ}\right)$ 534 higher than calculated for tilt angles below $14^{\circ}$, and slightly $535\left(<1^{\circ}\right)$ smaller for tilt angles above this threshold, with a 536 rapid increase of deviation when the film stops following 537 pure-3DGE growth mode for tilt above $20^{\circ}$ [Fig. 4(b)].

${ }_{538}$ The misorientation of $\mathrm{CeO}_{2}$ grains was tested using the ${ }_{539}$ XRD rocking curves measurements. For an increase of $\gamma$ 540 from $0^{\circ}$ to $19^{\circ}$ the FWHM of the rocking curves, as well as spread of the FHWM's from sample to sample, increased [see 541 Fig. 4(b), top curve]. When the growth mechanism turns to- 542 wards the standard mode $\left(\gamma=23^{\circ}\right)$, the width of the rocking 543 curve decreases.

The lattice constant of the $\mathrm{CeO}_{2} 3 \mathrm{DGE}$ films on TAS is 545 close to that of the films on (110) NGO substrate, 5.399-5.408 546 $\AA$. The strain and the FWHM of the rocking curves for the ${ }_{547}$ majority of the 3DGE films are significantly higher $(\sim 1 \%$ and 548 $\left.\sim 1.3^{\circ}\right)$ than that of the standard films $\left(\sim 0.15 \%\right.$ and $\sim 0.5^{\circ}$, see $\quad 549$ Table II). The FWHM of the peaks on the $\theta / 2 \theta$ scans is higher 550 than expected on the size evaluation, implying significant 551 inhomogeneity of the films similarly to the standard-oriented 552 films grown on (110) NGO. Small deviations from optimal 553 deposition conditions influences the 3DGE films properties in 554 the same way as that of the standard films: the lattice constant 555 increased and the strain in the film decreased with a decrease 556 of the oxygen partial pressure during deposition. The rocking 557 curve width showed no dependence on deposition conditions 558 in the studied range.

\section{YSZ on NGO TAS}

560

The typical rocking curves of the YSZ thin films on TAS 561 NGO are shown in Fig. 5. The Bragg diffraction angle for the 562 (400) peak of YSZ is very close to that of the (330) peak of the 563 NGO substrate, so a single measurement allows determination 564 of angular positions of both substrate and film SICPs. 565

Typical dependence of the YSZ film orientation on sub- 566 strate tilt angle is shown in Fig. 6(a). The tilt of the (100) 567 plane of YSZ follows the geometrical model, sine variant (2), 568 exceeding the calculated value in the angular range $5^{\circ}-12^{\circ} .{ }_{569}$ The deviation from the 3DGE model is much higher than 570 for $\mathrm{CeO}_{2} 3 \mathrm{DGE}$ films, reaching $+3^{\circ}$ for substrate tilt angles 571
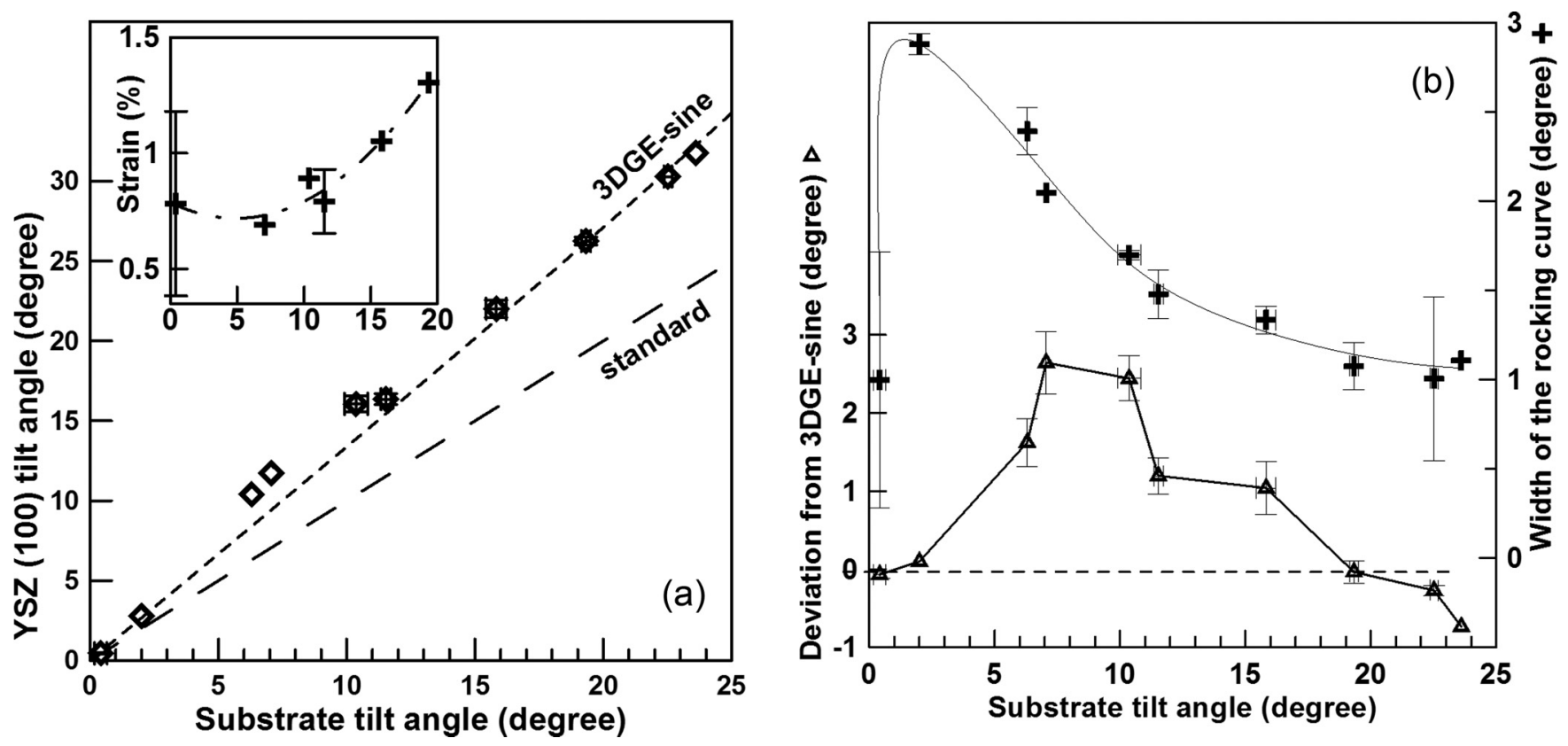

FIG. 6. Orientational relations of YSZ films on TAS NGO. (a) The film orientation follows the 3DGE-sine growth mode [Eq. (2)] at high substrate tilt angles and slightly exceeds the calculated value for $5^{\circ}-12^{\circ}$. Inset: Dependence of the strain in the film on the substrate tilt angle. (b) Deviation from calculated tilt angle (3DGE-sine model, triangles) shows maximum in the $7^{\circ}-10^{\circ}$ range, correlating with the width of the rocking curve dependence on tilt angle (crosses). Lines are given as guides for the eye. 
$5727^{\circ}-10^{\circ}$ [Fig. 6(b), bottom curve], but similarly decreases with 573 tilt angle, finally changing to negative values for tilt angles 574 above $20^{\circ}$. The FWHM of the rocking curve for the YSZ films 575 deposited at tilt angles close to zero shows a very high spread 576 from sample to sample [Fig. 6(b), top curve]. A change of the 577 substrate tilt angle to $\sim 2^{\circ}$ results in a huge rise of the grains 578 misorientation (the FWHM increases to $\sim 3^{\circ}$ ). The further 579 increase of the substrate tilt angle results in a gradual decrease 580 of FWHM with saturation at $\sim 1^{\circ}$ at high tilt angles.

581 The variation of the lattice parameter $\Delta d / d$ also shows 582 a very high spread from sample to sample at zero tilt angle 583 [see inset Fig. 6(a)]. Until $12^{\circ}$ it remains almost constant, 584 and increases only above $15^{\circ}$, when the deviation from the 585 calculated angle becomes small and the width of the rocking 586 curve saturates [Fig. 6(b)]. The strain and the width of the 587 rocking curve show clear anticorrelation.

588 The lattice constant of the YSZ films varied from 5.134 589 to $5.157 \AA$ (5.148 $\AA$ average, the measurement accuracy was 590 low, $\sim 0.007 \AA$ ), independently on the substrate tilt angle. The 591 deposition of YSZ films seem to be rather reproducible, no 592 significant changes in film orientation and structure could be 593 observed with small changes of deposition conditions. An 594 increase of thickness of the YSZ film (100 to $900 \AA$ ) leads 595 to a decrease of strain and a decrease of the rocking curve 596 width: with an increase of film thickness the film becomes 597 more homogeneous and more aligned.

\section{3. $\mathrm{BaZrO}_{3}$ on NGO TAS}

599 The expected growth mode of a perovskite BZO film on 600 a perovskite NGO substrate was standard, and for high $\left(24^{\circ}\right)$ 601 tilt angles this assumption proved to be correct. Surprisingly, 602 at a substrate tilt angle of $10^{\circ}$ the film orientation showed 603 excellent agreement with the geometrical model (Fig. 7). The 604 discrepancy from the calculated value (tangent model) is less 605 than $0.05^{\circ}$, and deviation from the standard orientational rela606 tions exceeds $0.8^{\circ}$. The width of the rocking curve increased 607 for high tilt angle (see inset Fig. 7), similarly to the $\mathrm{CeO}_{2}$ 608 3DGE films [Fig. 4(b)]. The strain in the films decreased 609 with tilt angle, anticorrelating to the rocking curve width. The 610 measured lattice constant was $4.199 \AA$, in good agreement 611 with the standard $4.2 \AA$ value, and did not depend on tilt angle.

\section{C. 3DGE growth in multilayer heterostructures}

613 All studied multilayer heterostructures on NGO TAS 614 showed 3DGE growth through the whole thickness of the 615 heterostructure, except when a chemical reaction took place 616 between the neighboring layers (YBCO over $\mathrm{CeO}_{2}$, YSZ, 617 or BZO). Even in these cases some part of the upper layer 618 showed 3DGE growth, especially at lowered deposition tem619 peratures.

\section{1. $\mathrm{CeO}_{2} / \mathrm{YSZ} / \mathrm{NGO}$}

621 A thin (20-100 $\mathrm{\AA}) \mathrm{CeO}_{2}$ layer is often introduced be622 tween the YSZ bottom layer and YBCO top layer to prevent 623 chemical interaction. A weak signal from the thin $\mathrm{CeO}_{2}$ layer 624 is hard to distinguish from a strong neighboring YSZ peak, 625 especially at small tilt angles. Still for some samples we 626 managed to determine the mutual orientation of the films in

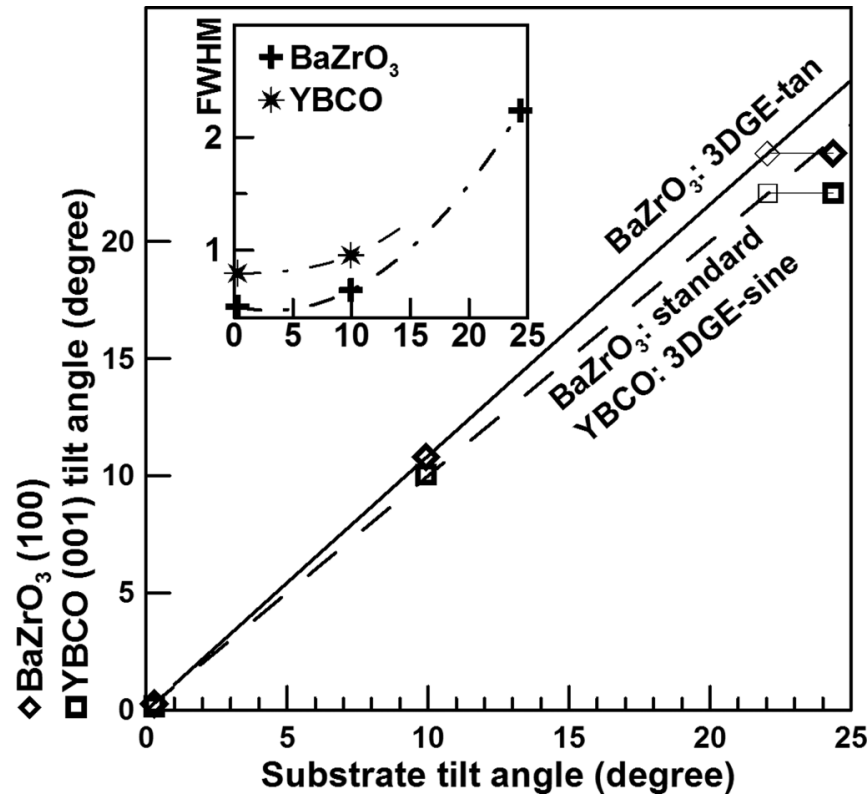

FIG. 7. Orientational relations in the $\mathrm{YBCO} / \mathrm{BZO}$ heterostructures on TAS NGO. Diamonds: BZO, squares: 3DGE part of the YBCO films. Thin symbols: Calculated positions if BZO kept 3DGE growth mode to $24^{\circ}$. Inset: Dependence of the width of the rocking curve of the BZO (200) peak (crosses) and YBCO (005) peak (stars) on the substrate tilt angle. The lines on the inset are guides for the eye.

a $\mathrm{YBCO} / \mathrm{CeO}_{2}(75 \AA)$ )/YSZ trilayer on NGO TAS (Fig. 8). 627 We expected standard epitaxial growth of a fluorite $\mathrm{CeO}_{2}$ film 628 over a fluorite YSZ bottom layer, with strictly parallel (100) ${ }_{629}$ planes in both layers. Instead, the 3DGE growth was observed 630 not only for the fluorite YSZ layer over the perovskite NGO 631 substrate, but also for the $\mathrm{CeO}_{2}$ film over the heterostructure 632 (Fig. 8). The tilt angle for the $\mathrm{CeO}_{2}$ layer is higher than that of ${ }_{633}$ the YSZ layer, in agreement with greater lattice constant (5.4 634

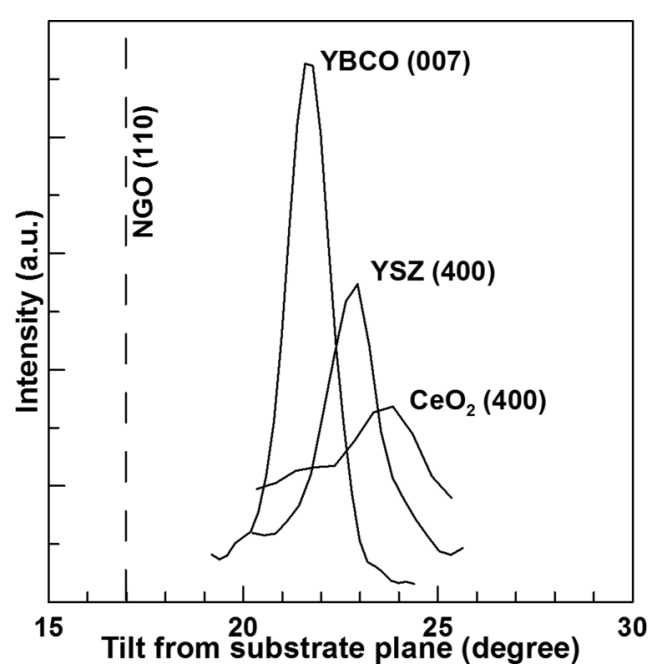

FIG. 8. Rocking curves of the (400) YSZ, (400) $\mathrm{CeO}_{2}$, and (007) YBCO peaks of the trilayer $\mathrm{YBCO} / \mathrm{CeO}_{2} / \mathrm{YSZ}$ heterostructure on TAS NGO. The substrate tilt angle is $16.9^{\circ}$. All layers follow the 3DGE growth mode. 


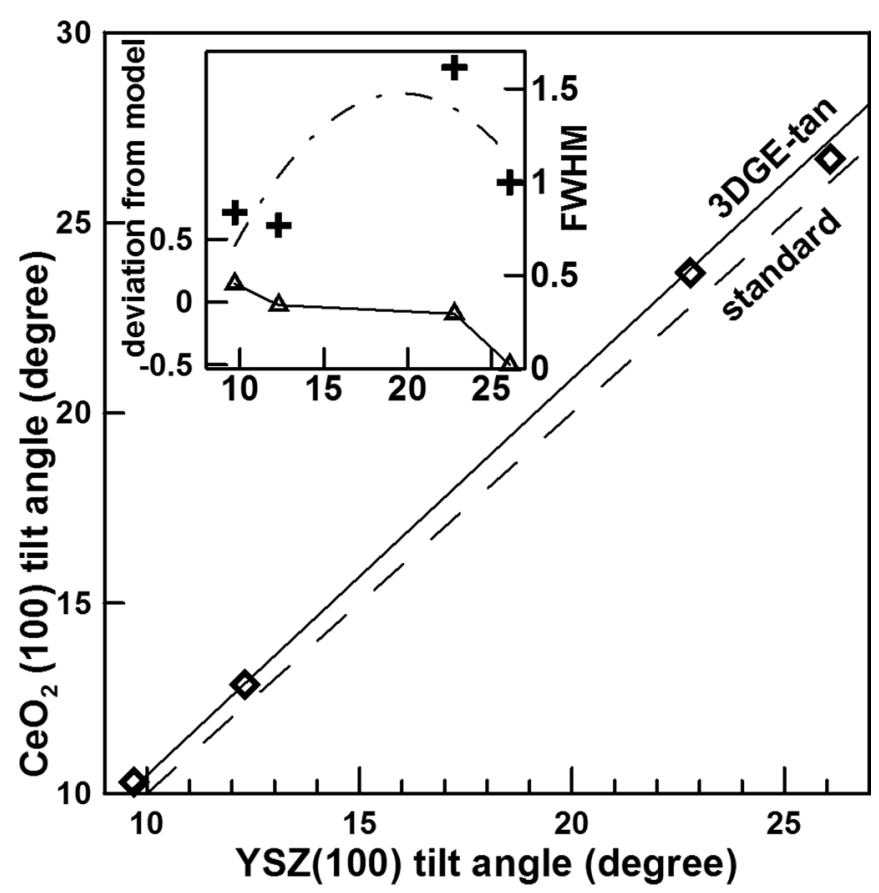

FIG. 9. Orientation of $\mathrm{CeO}_{2}$ films on 3DGE YSZ layers on TAS NGO. Inset: Dependence of the deviation from the 3DGE-tangent model (triangles) and the width of the rocking curve of the $\mathrm{CeO}_{2}$ (400) peak (crosses) on the substrate tilt angle. The lines are guides for the eye.

635 and $5.15 \AA$ ). The calculated and measured tilt angles match 636 well (Fig. 9). The tangent model (1) describes the angular 637 behavior better than the sine model (2), calculations are done 638 taking into account an excessive tilt of the YSZ layer for 639 tilt angles $5^{\circ}-12^{\circ}$ [Fig. 6(a)]. Similar to the $\mathrm{CeO}_{2}$ films on 640 bare NGO TAS the deviation from calculated value changes 641 from positive to negative at $\sim 12^{\circ}$ tilt of the underlaying layer 642 [compare Fig. 4(b) and inset Fig. 9]. At high tilts (above $64323^{\circ}$ for $\mathrm{CeO}_{2} /$ YSZ) a deviation towards the standard growth 644 mode is observed [Figs. 9 and 4(a)]. The width of the rocking 645 curve increases with tilt angle, but drops when the tilt changes 646 towards the standard orientation. We may conclude that all 647 orientational features of the 3DGE tangent mode growth of ${ }_{648} \mathrm{CeO}_{2}$ on TAS NGO are preserved on tilted-axes YSZ bottom 649 layer.

650 The measured lattice constant of the $\mathrm{CeO}_{2}$ interlayer is $6515.397 \pm 0.009 \AA$, somewhat smaller than the standard $5.4 \AA$ 652 value, or $5.404 \pm 0.004 \AA$ typical for $\mathrm{CeO}_{2}$ grown on NGO ${ }_{653} \mathrm{TAS}$ at the same deposition conditions. Incorporation of $\mathrm{Zr}$ 654 atoms into the $\mathrm{CeO}_{2}$ growing film may be the reason for this 655 difference.

\section{2. $\mathrm{YBCO} / \mathrm{CeO}_{2} / \mathrm{NGO}$}

657 The $\mathrm{YBCO}$ grains on a $\mathrm{CeO}_{2}$ layer showed either $c$ ori658 entation or 3DGE orientation; films with mixed orientation 659 were observed most commonly. The orientation of the YBCO 660 grains depended on tilt angle and deposition conditions, but 661 the most affecting factors are the properties of the underlaying $662 \mathrm{CeO}_{2}$ layer, set by $\mathrm{CeO}_{2}$ fabrication technique, and prepara663 tion of the $\mathrm{CeO}_{2} / \mathrm{NGO}$ sample to the YBCO film deposition.

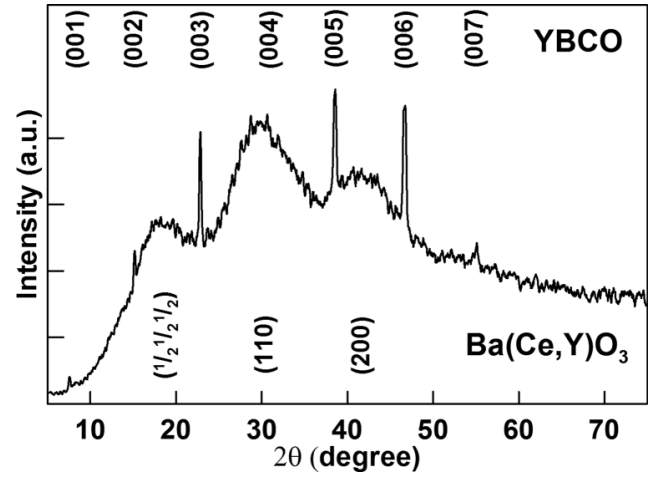

FIG. 10. X-ray $\theta / 2 \theta$ scan of the $\mathrm{YBCO} / \mathrm{CeO}_{2} / \mathrm{NGO}$ heterostructure along the substrate normal, nominal substrate tilt angle $22^{\circ}$. A set of broad peaks from polycrystalline $\mathrm{Ba}(\mathrm{Ce}, \mathrm{Y}) \mathrm{O}_{3}$ reaction layer can be detected on the scans, produced by small (5-15 $\AA$ ) crystallites.

The mechanisms of $c$-oriented YBCO film formation over 664 the $\mathrm{CeO}_{2}$ layer are similar to the growth processes of YBCO 665 films on the YSZ layers (see, e.g., [28,29]) and result from 666 interaction of $\mathrm{Ba}$ with $\mathrm{CeO}_{2}$ with formation of $\mathrm{a} \mathrm{Ba}(\mathrm{Ce}, \mathrm{Y}) \mathrm{O}_{3}{ }_{667}$ interlayer. An increase of thickness of such an interlayer leads 668 to secondary seeding of $\mathrm{Ba}(\mathrm{Ce}, \mathrm{Y}) \mathrm{O}_{3}$ grains with orientations 669 providing minimization of the surface energy. We observed 670 (100), (110), and (111) orientations of $\mathrm{Ba}(\mathrm{Ce}, \mathrm{Y}) \mathrm{O}_{3}$ grains 671 along the substrate plane (Fig. 10). The wide peaks in the 672 angular range $10^{\circ}-50^{\circ}$ corresponded to very small $(5-20 \AA) \quad{ }_{673}$ crystallites with lattice constant $\sim 4.31 \AA$. The YBCO films on 674 the $\mathrm{Ba}(\mathrm{Ce}, \mathrm{Y}) \mathrm{O}_{3}$ interlayer always showed $c$-oriented growth 675 (Fig. 10), sometimes mixed with the tilted grains. 676

The tilt angle of the tilted YBCO grains differed both from 677 the substrate tilt angle and the tilt angle of the $3 \mathrm{DGE} \mathrm{CeO}_{2} \quad 678$ layer (Fig. 8), and increased monotonously with the substrate 679 tilt angle, implying the 3DGE or some similar growth mech- 680 anism [Fig. 11(a), solid diamonds]. This effect was noticed 681 in [21], but no explanation was suggested. Assuming 3DGE 682 growth with a step height equal to $c / 3=3.933 \AA$ (on the early 683 stages of YBCO growth it tends to grow in the pseudocubic 684 form), we get the YBCO tilt angle very close to that of the 685 substrate (step height $3.864 \AA$ ), with a deviation below $1^{\circ}$ in ${ }_{686}$ the whole possible range of tilt angles. The actual deviation 687 from the substrate tilt angle is much higher [Fig. 11(a)]. 688

The explanation for the 3DGE growth with the ob- 689 served angles is also chemical interaction with forma- 690 tion of a very thin (not detected with XRD techniques) 691 $\mathrm{Ba}(\mathrm{Ce}, \mathrm{Y}) \mathrm{O}_{3}$ layer between $\mathrm{CeO}_{2}$ and $\mathrm{YBCO}$ films. This 692 layer is strictly aligned with the underlying $\mathrm{CeO}_{2}$ film, 693 (100) $\mathrm{Ba}(\mathrm{Ce}, \mathrm{Y}) \mathrm{O}_{3} \|(100) \mathrm{CeO}_{2}$, and the tilt angle of the 694 YBCO film is determined not by step height of $\mathrm{CeO}_{2}$ layer 695 but that of $\mathrm{Ba}(\mathrm{Ce}, \mathrm{Y}) \mathrm{O}_{3}$. From the atomistic point of view 696 we may assume chemical bonding of the surface $\mathrm{CeO}$ layer ${ }_{697}$ with the incoming $\mathrm{Ba}$ atoms, with formation of a half of a 698 perovskite $\mathrm{BaCeO}_{3}$ cell, providing out-of-plane lattice con- 699 stant (and, consequently, terrace step height) corresponding to 700 $\mathrm{BaCeO}_{3}$, not $\mathrm{CeO}_{2}$. We note that 1/2- or 1/3-lattice constant 701 as the height of the surface step was already mentioned before 702 $[15,17]$. The agreement with the 3DGE-sine model assuming 703 $\mathrm{BaCeO}_{3}$ step height (4.31 $\AA$ measured for small crystallites) 704 

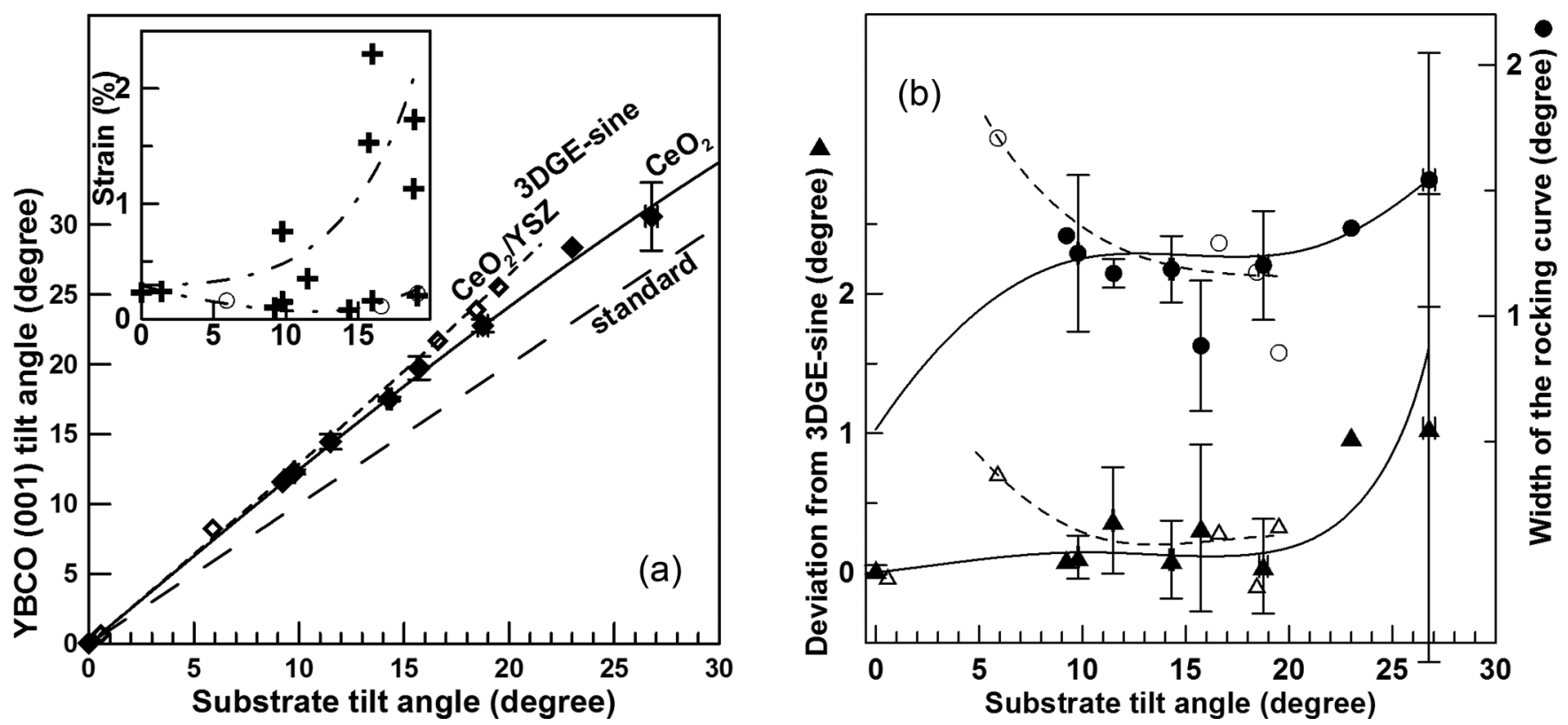

FIG. 11. Orientational relations of $\mathrm{YBCO}$ films on $\mathrm{CeO}_{2}$ layers and on $\mathrm{CeO}_{2} / \mathrm{YSZ}$ bilayers on TAS NGO. (a) The YBCO film orientation follows the 3DGE-sine growth mode [Eq. (2)] both for films grown on a single $\mathrm{CeO}_{2}$ layer (solid diamonds, solid line) and on $\mathrm{CeO} / \mathrm{YSZ}$

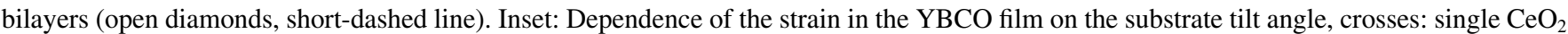
layers, circles: $\mathrm{CeO}_{2}$ /YSZ bilayers. (b) Absolute deviation from calculated tilt angle (3DGE-sine model, triangles) correlates with the width of the rocking curve (circles). Solid symbols correspond to the $\mathrm{YBCO}$ films on single $\mathrm{CeO}_{2}$ layers, open symbols $\mathrm{YBCO}_{\text {films on } \mathrm{CeO}} / \mathrm{YSZ}$ bilayers. The lines are given as guides for the eye.

705 is impressive up to $27^{\circ}$ [Fig. 11(a), solid diamonds]. Note 706 that down-bending of the calculated dependence [solid line 707 Fig. 11(a)] is determined by the tangent dependence of the tilt 708 of the $\mathrm{CeO}_{2}$ layer [Fig. 4(a)]. Measured data for the $\mathrm{CeO}_{2}$ 709 films at substrate tilt angles above $23^{\circ}$ are absent, so the 710 curve Fig. 11(a) in this range was calculated assuming tangent 711 dependence for $\mathrm{CeO}_{2}$ and sine formula for $\mathrm{YBCO}$ over the 712 intermittent $\mathrm{Ba}(\mathrm{Ce}, \mathrm{Y}) \mathrm{O}_{3}$ layer.

713 The average deviation of the YBCO film orientation from 714 the calculated value remained small $\left(\sim 0.2^{\circ}\right)$ until $\sim 20^{\circ}$, and 715 rapidly increased for tilt angles above $20^{\circ}$ [Fig. 11(b), solid 716 triangles]. The FWHM of the rocking curves repeated this 717 dependence [Fig. 11(b), solid circles]. The lattice constant $c$ of 718 the YBCO films was almost constant $11.684 \pm 0.007 \AA$ for all 719 tilt angles below $20^{\circ}$. Precise determination of the lattice con720 stants for higher angles was complicated due to the limitations 721 of the applied asymmetric geometry of the X-ray diffraction. 722 The strain $\Delta d / d$ was estimated for the same angular range ${ }_{723} 0^{\circ}-20^{\circ}$; the films can be divided into two groups, with very 724 low strain below $0.2 \%$, and with strain increasing with tilt 725 angle to $1 \%$ and more [see inset Fig. 11(a)]. The increasing 726 dependence resembles strain behavior in the 3DGE YSZ films 727 on TAS NGO.

\section{8 \\ 3. $\mathrm{YBCO} / \mathrm{CeO}_{2} / \mathrm{YSZ} / \mathrm{NGO}$}

729 The trilayer $\mathrm{YBCO} / \mathrm{CeO}_{2} / \mathrm{YSZ}$ structures were not stud730 ied extensively due to the low $(30-80 \AA)$ thickness chosen 731 for the $\mathrm{CeO}_{2}$ layers for the planned experiment. Still some 732 samples allowed measurements for all three layers (Figs. 8 733 and 9).
The YBCO films on the $\mathrm{CeO}_{2} / \mathrm{YSZ}$ bilayer show the ${ }_{734}$ same orientational behavior as on the single $\mathrm{CeO}_{2}$ layers: 735 $c$-oriented, 3DGE-oriented, and mixed-orientation films were 736 formed depending on conditions for the chemical reaction 737 with formation of a $\mathrm{Ba}(\mathrm{Ce}, \mathrm{Y}) \mathrm{O}_{3}$ layer. The properties of the 738 3DGE grains are shown with open symbols in Fig. 11.

The tilt of the YBCO film [Fig. 11(a), open diamonds] 740 is described by the same sine model as in the bilayer case. 741 Introduction of the YSZ layer with sine 3DGE dependence 742 below a $\mathrm{CeO}_{2}$ layer with tangent dependence results in a sig- 743 nificant difference $\left(0.3^{\circ}-1.0^{\circ}\right)$ between the measured values 744 in the high-angle range, clearly distinguished with the applied 745 XRD techniques. The deviation from the calculated value is 746 slightly higher than for the YBCO 3DGE films on a single 747 $\mathrm{CeO}_{2}$ layer [Fig. 11(b), open triangles], and increases at low 748 tilt angles $\left(5^{\circ}-8^{\circ}\right)$. Unfortunately, there is no available data 749 for YBCO 3DGE films on a single layer for this angular 750 range. The YSZ 3DGE films showed a similar increase of 751 deviation for the $5^{\circ}-10^{\circ}$ range [Fig. 6(b)]. The width of the 752 rocking curve correlated well with the absolute deviation 753 value, similarly to the YBCO 3DGE films on a single $\mathrm{CeO}_{2} 754$ layer [Fig. 11(b), closed symbols]. The FWHM of the rocking 755 curve in the angular range $15^{\circ}-20^{\circ}$ does not differ much from 756 the measured values for YBCO 3DGE films on a single $\mathrm{CeO}_{2}{ }_{757}$ layer.

The lattice constant of the 3DGE YBCO films on the 759 $\mathrm{CeO}_{2} / \mathrm{YSZ}$ bilayers is the same as for the 3DGE YBCO films 760 on single $\mathrm{CeO}_{2}$ layers: $11.684 \pm 0.005 \AA$, and also shows 761 no angular dependence. The evaluated strain is low for all 762 studied films, less than $0.25 \%$, so all the YBCO films on 763 bilayers belong to the low-strain group of samples [see inset 764 Fig. 11(a)]. 
To prove $\mathrm{YBCO}$ growth mechanisms on $\mathrm{BaCeO}_{3}$ we fabri-

801 standard-oriented BZO grains on the rocking curves, being out 802 of the scan range.

803 The main part of the $\mathrm{YBCO} / \mathrm{BZO}$ heterostructure showed 804 the same properties as $\mathrm{YBCO} / \mathrm{Ba}(\mathrm{Ce}, \mathrm{Y}) \mathrm{O}_{3}$ layer on $3 \mathrm{DGE}$ $805 \mathrm{CeO}_{2}$ films. The main part of the $\mathrm{BZO}$ layer was growing 806 (100), (110), and (111) oriented, with $c$-oriented YBCO grow807 ing over these grains. Some part of the BZO layer followed the 808 3DGE-sine growth mode on tilted YSZ layer with tilt angle 809 of $19.28^{\circ}$ (calculated value $19.35^{\circ}$ ). YBCO growth on these 810 3DGE BZO grains was not detected, neither in standard nor 811 in 3DGE orientation.

\section{DISCUSSION}

${ }_{813}$ The growth of films with significant tilt of SICP from habit 814 planes of the tilted-axes substrate are usually considered as 815 rare and, in some sense, exceptions from the general rule of 816 standard growth mode. Our results show that, in fact, for all817 metaloxide heterostructures the 3DGE growth mechanism is 818 more common than standard, especially for the angular range 819 below $20^{\circ}$.
820

821 822

\section{A. Epitaxial issues}

To start discussing the 3DGE growth mechanism we should first revert to the question of epitaxy. In the very beginning this term was used when the structure of the film ${ }^{823}$ repeated the structure of the substrate. Rigorously only the 824 films of the same material as the substrate, but with different 825 dopant or with different level of doping, may be considered 826 epitaxial. This term sometimes was also used to describe ${ }_{827}$ heterostructures in which the material of the film had the same ${ }_{828}$ lattice structure as the substrate, and the lattice constants did 829 not differ much. Later the term epitaxy was used when a strict 830 relation could be established between crystallographic planes 831 and directions of the film and crystallographic planes and ${ }_{832}$ directions of the substrate. These films were also known as 833 oriented films. The orientational relations with the substrate 834 for some of the oriented films were set by the atomic structure 835 of the substrate surface, and this class of film inherited the 836 name of epitaxial films, while the films for which some of ${ }_{837}$ the orientational relations are set by film surface retained the 838 name of oriented films.

The films presented in this study show no parallelism 840 between some SICPs in the film and in the substrate, only 841 the tilt axis is bonding the film and substrate lattice in a strict ${ }_{842}$ way. At the same time, the general orientation of the film is 843 set not by the surface of the film, but by the microstructure 844 of the substrate surface. The changes of the microstructure, 845 in particular, the distance between the edges of the terraces, 846 result in a change of the film orientation, in agreement with a 847 strict mathematical relation. From this point of view the films 848 are epitaxial, not just oriented.

The term graphoepitaxy, if it had been suggested in the 850 very beginning of the fabrication of epitaxial films, would ${ }_{851}$ have been considered as an oxymoron, something like a "dry ${ }_{852}$ liquid." The orientation of the graphoepitaxial films was set ${ }^{853}$ not by the atomic structure of the substrate, but by the macro- ${ }^{854}$ scopic structures of the surface of the substrate. Actually, the 855 first graphoepitaxial films were fabricated from a material 856 with totally different crystal structure from the structure of ${ }_{857}$ the substrate, making application of the term of "epitaxy" in 858 the strict meaning absolutely impossible. Still the term settled, 859 and at the moment the "graphoepitaxial" oriented films are 860 fabricated, discussed, and categorized in different types (see, 861 for example [30]).

Our films are (i) oriented and (ii) this orientation is set 863 by the surface features of the substrate, i.e., they can be 864 considered as graphoepitaxial. At the same time, the surface 865 features that set the orientational relations are determined 866 by the crystal structure of the substrate, and this makes our ${ }_{867}$ films epitaxial in the modern meaning of the term. Thus, 868 the films grown by the 3DGE mechanism are epitaxial and 869 graphoepitaxial at the same time.

We would like to emphasize a third point: the bonding of 871 the lattices of the film and the substrate is realized in three 872 orthogonal directions: the tilt axis, the length of the terrace, 873 and the out-of-plane lattice constants set the orientational re- 874 lations in a unique way. Moreover, the translational distances 875 in all three directions are important for the film orientation, 876 as well as matching (and mismatching) of these distances in 877 the film and the substrate. This makes the discussed growth 878 mechanism essentially three dimensional, and justifies the 879 proposed name "three-dimensional graphoepitaxial," 3DGE, 880 mechanism. 


\section{B. Growth modes and models}

Disregarding the complicated microstructural mechanisms 84 of formation of tilt in the films on TAS [11-13], we may 885 identify two simplified mechanisms of formation of the 3DGE 886 films: the overgrowth mechanism [15], and the simultaneous 887 seeding mechanism [16].

888 The first one suggests seeding of the film near the edge889 terrace joints, and growth of these from the terrace edge. 890 When the growing layer reaches the end of the terrace, it 891 encounters a different height of the surface that it should 892 overgrow, and accommodates this difference [Fig. 1(b)]. The 893 resulting angle depends on the length of the terrace $d_{t}$ and 894 simple considerations result in the tangent model (1). This 895 growth mode corresponds to the graphoepitaxy of cases 2 and 8963 in Fig. 1(b) in [30].

897 The second simplified model implies simultaneous seeding 898 of the film on neighboring "seeding knots" in the edge-terrace 899 joints [Fig. 1(c)]. In this case orientation of the film is formed 900 in the same way as for the standard growth mode, because 901 the seeding knots mimic the atom position in the atom-on902 atom epitaxial growth. The tilt angle of the film depends on 903 the distance between the seeding knots $d_{s}$, and the resulting 904 model follows a sine dependence (2).

905 The two models hardly can be distinguished at tilt angles 906 below $10^{\circ}$, so previous studies never faced a necessity to 907 choose between these two mechanisms.

908 The first model seems to be more probable for small tilt 909 angles, because the lattice of the growing layer should settle 910 well before overgrowth would change the tilt angle. The 911 demand to "settle" the structure of the growing layer means 912 that at high angles this mechanism is improbable. Assuming 913 two unit cells as a smallest "settled" seed, we get the limit for 914 the tangent mode growth:

$$
\gamma_{t}<\arctan \left[c_{s} /\left(2 a_{f}\right)\right],
$$

915 where $a_{f}$ is the film unit cell size along the growth direction. ${ }_{916}$ For $\mathrm{CeO}_{2}$ growth on $\mathrm{NGO}$ this formula gives $\gamma_{t}=19.7^{\circ}$, 917 and, indeed, at angles above $20^{\circ}$ the $\mathrm{CeO}_{2}$ film deviates 918 towards the standard growth mode [Fig. 4(a)]. $\mathrm{CeO}_{2}$ depo919 sition on YSZ formula (4) gives $25.5^{\circ}$, and the observed 920 deviation starts at $24^{\circ}-25^{\circ}$ (Fig. 9). The change of the growth 921 mechanism to standard for BZO on $\mathrm{NGO}$ at $24^{\circ}$ (Fig. 7, 922 diamonds) also may be a result of limited angular range of 923 the tangent growth mode $\left(\gamma_{t}=24.7^{\circ}\right)$. For YSZ on NGO the 924 limiting angle is $20.6^{\circ}$, but we cannot see a distinct trend 925 towards standard growth mode even for $\sim 23.5^{\circ}$. Note that the 926 sine model provides a better fit for 3DGE growth of YSZ. 927 Similarly no pronounced deviation towards standard growth 928 at high angles was observed for $\mathrm{YBCO}$ on $\mathrm{CeO}_{2}$, another pair 929 of materials better described by the sine formula (Fig. 11).

${ }_{930}$ The second simplified mechanism, simultaneous seeding, 931 to the contrary, is promoted by a small distance between the 932 seeding knots, and would be favorable at high tilt angles. At 933 small tilt angles the seeding would be more efficient for closer 934 placed seeding knots, so, taking into account a certain spread 935 of the distance $d_{s}$ along the substrate surface, we may expect 936 slightly higher average tilt angles compared to the calculated 937 value. This is exactly what happens for YSZ on NGO (Fig. 6), 938 and for $\mathrm{YBCO}$ on $\mathrm{CeO}_{2}$ [Fig. 11(b), open triangles].

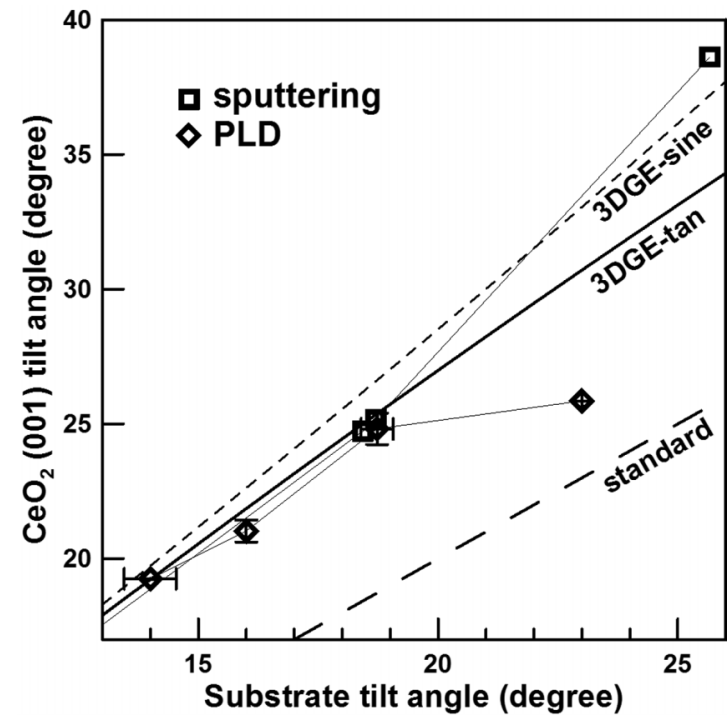

FIG. 12. Comparison of tilt of $\mathrm{CeO}_{2}$ films on NGO TAS deposited by PLD (diamonds) and rf sputtering (squares, [19,20]) at high substrate tilt angles.

Do we really observe two different 3DGE growth modes, 9з9 or is it just a measurement error? The data in Fig. 11(a) seem 940 to prove the presence of two different modes: the YBCO films 941 on a single $\mathrm{CeO}_{2}$ layer and on a $\mathrm{CeO}_{2} / \mathrm{YSZ}$ bilayer show dif- 942 ferent angular dependence in the range $15^{\circ}-20^{\circ}$ because a sin- 943 gle $\mathrm{CeO}_{2}$ layer fabricated by PLD follows the tangent mode, 944 while a YSZ layer below $\mathrm{CeO}_{2}$ demonstrates sine dependence. 945 Comparison of $\mathrm{CeO}_{2}$ films fabricated by different techniques 946 also seem to prove the existence of two different growth 947 modes (Fig. 12): the tilt of the PLD films at angles above $20^{\circ}{ }_{948}$ downturns towards the standard growth mode, while for the 949 films deposited with sputtering techniques [19,20] the tilt still 950 follows the 3DGE mechanism, sine dependence.

The data on growth modes for different film-substrate combinations are gathered in Table III. We could not unam- 953 biguously determine the factors that promote the overgrowth 954 mechanism or the simultaneous seeding mechanism. Still, as 955 much as we may conclude from the data in Table III, the com- 956 pressive strain promotes the overgrowth mechanism, while 957 films with tensile strain introduced by the underlying layer 958 tend to follow the simultaneous seeding mode. Compressive 959 strain, in the case of cubic film and substrate lattices, and 960 equality of step height and lattice constant, corresponds to 961 $c_{f}>c_{s}$ and increased tilt angle of the top layer compared to 962 the tilt angle of the bottom layer. Tensile strain, with the same 963 assumptions, corresponds to a decreased tilt angle of the top 964 layer, so the tangent mode seems to be typical for the increase 965 of the tilt angle due to the 3DGE growth mechanism, while the 966 sine mode for the decrease of the tilt angle. This is not so for 967 more complicated film-substrate matching, like YSZ/NGO, 968 when tensile strain (and simultaneous seeding mechanism) is 969 introduced into the film due to the $45^{\circ}$ axes tilt in the habit 970 plane (110) NGO, but the film tilt angle $\gamma^{\prime}$ increases because 971 the step height of YSZ is higher than that of NGO (Fig. 6). $\quad 972$

The dependencies of the critical parameters on the lattice 973 mismatch seem to confirm this observation: for example, 974 
TABLE III. Observation of sine and tangent growth modes for different film-substrate combinations.

\begin{tabular}{|c|c|c|c|c|c|}
\hline Film/substrate & Lattice mismatch $^{\mathrm{a}}(\%)$ & Growth mode & $\gamma_{t}$, measured/calculated $(\mathrm{deg})$ & Excessive tilt, value/range (deg) & Comment \\
\hline $\mathrm{BZO} / \mathrm{NGO}$ & $-8.7--8.8$ & tangent $^{\mathrm{b}}$ & $(<24) / 24.7$ & $\mathrm{n} / \mathrm{a}$ & \\
\hline $\mathrm{CeO}_{2} / \mathrm{YSZ}$ & -5.0 & tangent & $\sim 24.5 / 25.5$ & $\mathrm{n} / \mathrm{a}$ & \\
\hline $\mathrm{CeO}_{2} / \mathrm{NGO}$ & $+1.0-+1.1$ & $\begin{array}{l}\text { tangent } \\
\text { sine }\end{array}$ & $\begin{array}{c}20 / 19.7 \\
\text { n/a }\end{array}$ & $\begin{array}{c}\text { n/a } \\
\text { no data }\end{array}$ & $\begin{array}{l}\text { by PLD } \\
\text { by rf sputtering }\end{array}$ \\
\hline $\mathrm{YBCO} / \mathrm{CeO}_{2}$ & $-1.8--0.1$ & sine & $\mathrm{n} / \mathrm{a}$ & $0.7 / 6$ & \\
\hline YSZ/NGO & $+5.7-+5.8$ & sine & $\mathrm{n} / \mathrm{a}$ & $2.5 /(6-10)$ & \\
\hline $\mathrm{YBCO} / \mathrm{BZO}$ & $+7.4-+8.9$ & $\operatorname{sine}^{\mathrm{b}}$ & $\mathrm{n} / \mathrm{a}$ & $(0.03 / 10)$ & \\
\hline $\mathrm{Ba}(\mathrm{Y}, \mathrm{Zr}) \mathrm{O}_{3} / \mathrm{YSZ}$ & $+11.5^{\mathrm{c}}$ & $\sin e^{b}$ & $\mathrm{n} / \mathrm{a}$ & no data & \\
\hline
\end{tabular}

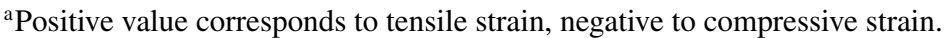

${ }^{\mathrm{b}}$ Insufficient data for a reliable conclusion.

${ }^{\mathrm{c}} \mathrm{No} 45^{\circ}$ in-plane rotation.

975 the deviation towards the standard mode for the overgrowth 976 mechanism seems to start at smaller angles for higher com977 pressive strain, as seen from the $\mathrm{BZO} / \mathrm{NGO}, \mathrm{CeO}_{2} / \mathrm{YSZ}$, and 978 the $\mathrm{CeO}_{2} / \mathrm{NGO}$ pairs. The excessive tilt at small tilt angles 979 seems to increase with an increase of the tensile lattice mis980 match, when we compare the $\mathrm{YBCO} / \mathrm{CeO}_{2}$ and $\mathrm{YSZ} / \mathrm{NGO}$ 981 combinations (Table III).

982 Two rows of Table III with small mismatches $\sim 1 \%$ contra983 dict to this suggested rule: $\mathrm{YBCO} / \mathrm{CeO}_{2}$ (small compressive 984 strain, sine dependence) and $\mathrm{CeO}_{2} / \mathrm{NGO}$ (small tensile strain, 985 tangent dependence). If we suppose that the $\mathrm{CeO}_{2}$ lattice 986 constant is $\sim 1.5 \%$ higher than measured, we remove this 987 discrepancy: the lattice mismatch for $\mathrm{CeO}_{2} / \mathrm{NGO}$ becomes 988 weakly compressive $(\sim-0.5 \%)$, and for $\mathrm{YBCO} / \mathrm{CeO}_{2}$, 989 weakly tensile $(\sim+0.6 \%)$, in good agreement with the 990 other data. The thermal expansion coefficients cannot ac991 count for such corrections, being almost the same for all 992 three materials [NGO : (4.5-9.0) $\times 10^{-6} \mathrm{~K}^{-1}$ from differ993 ent references, $\mathrm{CeO}_{2}$ : $(8.5-9.5) \times 10^{-6} \mathrm{~K}^{-1}$, and $\mathrm{YBCO}$ : $994(11-13) \times 10^{-6} \mathrm{~K}^{-1}$ ]. The increase of the $\mathrm{CeO}_{2}$ lattice con995 stant during deposition can happen as a result of incomplete 996 oxygenation (see discussion of oxygen removal from and 997 incorporation to, for example, in [31]), and $1.5 \%$ is not the 998 highest possible expansion. The missing oxygen could be 999 incorporated into the $\mathrm{CeO}_{2}$ layer immediately after the depo1000 sition, shrinking the lattice constant to the observed value.

1001 For $\mathrm{CeO}_{2}$ /NGO both mechanisms were observed for dif1002 ferent deposition techniques. We note that for e-beam evap1003 oration and $\mathrm{rf}$ sputtering the deposition rate is small, so 1004 the $\mathrm{CeO}_{2}$ film grows completely oxygenated, while for PLD 1005 a certain amount of oxygen vacancies is generated in the 1006 growing film, expanding the $\mathrm{CeO}_{2}$ lattice. Tensile strain for 1007 the completely oxygenated $\mathrm{CeO}_{2}$ films would account for sine 1008 mode for the e-beam evaporated and rf-sputtered films, while 1009 an increase of lattice constant by more than $1 \%$ during PLD 1010 would result in a compressive strain and tangent growth mode.

\section{$1011 \quad$ C. Orientational features}

1012 The width of the rocking curve is a parameter that sheds 1013 light on the peculiarities of the 3DGE film formation. The 1014 misorientation of the grains strongly depends on the seeding 1015 mechanism, and differences in rocking curve width depen- dence on angle imply formation of 3DGE films in different ${ }_{1016}$ ways.

A comparison of properties of $\mathrm{CeO}_{2}$ films on NGO TAS ${ }_{1018}$ [Fig. 4(b)] and on YSZ 3DGE layers on NGO TAS (inset 1019 Fig. 9) show clear similarities. The deviation from the cal- 1020 culated curve slowly decreases from small-positive to small- ${ }_{1021}$ negative values until the threshold angle $\gamma_{t}$, when negative 1022 deviation rapidly increases. This dependence is accompanied ${ }_{1023}$ by an increase of the width of the rocking curve until the 1024 same threshold angle, after which it drops to smaller level. 1025 Both sets of data can be brought to the same scale by plotting ${ }_{1026}$ the dependence on the film tilt angle instead of using the ${ }_{1027}$ substrate tilt angle, and by normalizing the deviation from the ${ }_{1028}$ calculated value by division on the inclination $\left(\gamma^{\prime}-\gamma\right)$ due ${ }_{1029}$ to the 3DGE growth mechanism. This transformation makes 1030 visible the identity of the two dependencies [Fig. 13(a)]. ${ }_{1031}$ Unfortunately, we have not enough data in the range $0^{\circ}-10^{\circ}{ }_{1032}$ to present the complete dependence of the deviation from the ${ }_{1033}$ geometrical model (1).

The reasons for the misorientation of the grains of the ${ }_{1035}$ film were presented in [15]. The terraces on the substrate ${ }_{1036}$ surface are not equal, so a certain spread of the orientation ${ }_{1037}$ of the grains after overgrowth of the next step is present ${ }_{1038}$ from the beginning, and strongly depends on the size of 1039 the grains of the film. If the grains are smaller than the 1040 typical terrace length $d_{t}$, the grains would grow following the 1041 standard growth mode. This effect is clearly demonstrated in 1042 [17], where the change of the growth mode to standard at low 1043 deposition temperature is a result of a decrease of the size ${ }_{1044}$ of the $\mathrm{CeO}_{2}$ grains. The combination of a distribution of size 1045 of the grains and a distribution of the length of the terraces 1046 results in a distribution of the orientation of the grains starting 1047 from the standard mode and until film tilt angles exceeds the 1048 calculated $\gamma_{t}$ using the geometrical model. The spread of the 1049 orientation of the grains will increase when step bunching 1050 starts on the substrate surface, with formation of steps with 1051 height 2, 3, and more times the ordinary height $c_{s}$. For the 1052 typical perovskite substrates step bunching starts at $\sim 10^{\circ},{ }_{1053}$ depending on preparation conditions [5], and we observe an 1054 increase of the width of the rocking curve at $\sim 13^{\circ}$ [Fig. 13(a)]. 1055 The rapid decrease of the film tilt angle towards the standard 1056 relations at angles above the threshold $\gamma_{t}$ may be a result of ${ }_{1057}$ very small lengths of terraces, so that the overgrowth happens 1058 

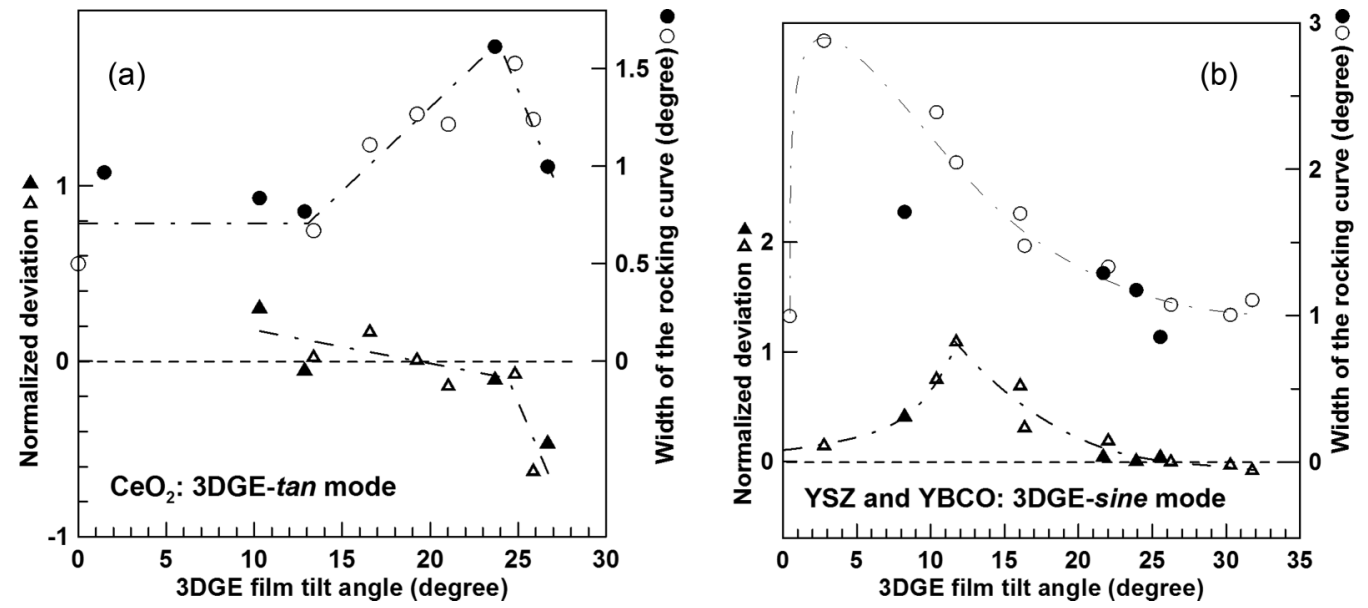

FIG. 13. Orientational properties of the 3DGE films with different growth mechanism. The overgrowth mechanism (a) shows an increase of the rocking curve width accompanied by a slow decrease of the deviation from positive to negative values until after the threshold angle FWHM of rocking curve decreases and the negative deviation rapidly increases. Open symbols: $\mathrm{CeO}_{2}$ films on NGO TAS, solid symbols: $\mathrm{CeO}_{2}$ films on 3DGE YSZ film on NGO TAS. The simultaneous seeding mechanism (b) shows a gradual decrease of width of the rocking curve from very high level obtained at very low tilt angles. The deviation shows a peak at $\sim 12^{\circ}$. Note that the YBCO films (solid symbols) were deposited over a YSZ layer (with a $\mathrm{CeO}_{2}$ interlayer), so the observed agreement between YBCO and YSZ (open symbols) data may be explained as inheritance of the properties of the bottom layer. The lines are given as guides for the eye.

1059 over two or more steps and the geometrical model (1) becomes 1060 invalid.

1061 The dependencies for the sine model are completely dif1062 ferent [Fig. 13(b)]. Even a small tilt of the substrate SICPs 1063 results in a very broad rocking curve of the film. A very high 1064 spread of the width of the rocking curve of the YSZ films on 1065 a standard-oriented substrate [Fig. 6(b)] implies realization of 1066 the 3DGE-sine mechanism even at very low tilt angles that are 1067 always present on the surface of a standard-oriented substrate 1068 due to inhomogeneous polishing. The rocking curves become 1069 more and more narrow with an increase of the substrate tilt 1070 until the FWHM of the rocking curves saturates at $\sim 20^{\circ}$ 1071 (film tilt angle $\sim 25^{\circ}$ ). The deviation from the calculated value 1072 at this angle changes from positive to negative, but remains 1073 small-no turn-down to the standard epitaxial relations was 1074 observed for the 3DGE-sine growth mode in the whole studied 1075 range, until $\sim 35^{\circ}$. The highest deviation is observed at $5^{\circ}-$ $107610^{\circ}$ [film tilt $\sim 12^{\circ}$, Fig. 13(b)]. This dependence is well 1077 explained by the simultaneous seeding mechanism. At high 1078 tilt angles the seeding knots on the substrate surface form 1079 a dense network, providing good conditions for seeding of 1080 the grains with the exact tilt angle, determined by condition 1081 (2). Both deviation and misorientation of the grains is small. 1082 A decrease of the substrate tilt angle increases the distances 1083 between the seeding knots and, hence, increases the width 1084 of the distribution of $d_{s}$. An immediate consequence is the 1085 increased misorientation of the grains of the film and, hence, 1086 the width of the rocking curve. This tendency remains the 1087 same until very small angles, when the standard growth mode 1088 becomes dominant. Another consequence of the increased 1089 distance between the seeding knots is a shift of the distribution 1090 of the orientation of the grains towards higher tilt angles. 1091 The reason is a higher probability of seeding of a grain of 1092 a certain orientation when the distance between the seeding 1093 knots is smaller, i.e., with higher tilt angle. This effect is 1094 less influential when all distances $d_{s}$ become long and the probability of seeding becomes even. For the YSZ films this 1095 happens for substrate tilt angles below $7^{\circ}$ (film tilt angle 1096 $\sim 12^{\circ}$ ).

YBCO on $\mathrm{CeO}_{2}$ seems to grow in the 3DGE-sine mode ${ }_{1098}$ also (Fig. 11), but we could not observe the same effects 1099 as for YSZ. The probable reason is the inheritance of the 1100 tangent mode properties of the $\mathrm{CeO}_{2}$ layer. The YBCO films 1101 on a $\mathrm{CeO}_{2} / \mathrm{YSZ}$ bilayer shows dependencies similar to that 1102 of YSZ [solid symbols in Fig. 13(b)] but, again, it may be a 1103 consequence of the sine growth mode of the YSZ layer below. ${ }_{1104}$ The parameters of the YBCO films on a $\mathrm{CeO}_{2} / \mathrm{YSZ}$ bilayer 1105 after normalization lies along the same lines as that of YSZ 1106 3DGE films [Fig. 13(b)].

An excess of film tilt $\left(0.5^{\circ}-1^{\circ}\right)$ over the calculated using 1108 the geometrical model was observed for $\mathrm{CeO}_{2}$ on $\mathrm{Ni}$ in 1109 [17]. The angular range for this excessive tilt increased with 1110 deposition temperature $T_{D}$ from $1^{\circ}-11^{\circ}$ at $785{ }^{\circ} \mathrm{C}$, to $1^{\circ}-15^{\circ}{ }_{1111}$ at $700{ }^{\circ} \mathrm{C}$, and $2^{\circ}-15^{\circ}$ at $600{ }^{\circ} \mathrm{C}$ (the upper angular limit 1112 for the excessive tilt is observed only for the highest $T_{D}$ ). ${ }_{1113}$ Assuming simultaneous seeding over a step, we get higher ${ }_{1114}$ probability of seeding on long distances $d_{S}$ with increased $T_{D}, \quad{ }_{1115}$ and, hence, a shift of the excessive tilt range to smaller tilt ${ }_{1116}$ angles.

\section{Effect of surface features}

The 3DGE growth mechanism essentially depends on the 1119 morphology of the substrate. Rigorous preparation of the 1120 substrate surface by chemical etching and annealing at high 1121 temperature (surface reconstruction) results in a regular se- 1122 quence of uniform steps one-unit-cell high. Such a substrate ${ }_{1123}$ provides the most refined conditions for testing certain growth 1124 effects and mechanisms.

Unfortunately, our depositions were performed on the sub- 1126 strates with no special treatment to form the SICP-faceted 1127 growth steps. The substrate showed a very smooth surface, 1128 

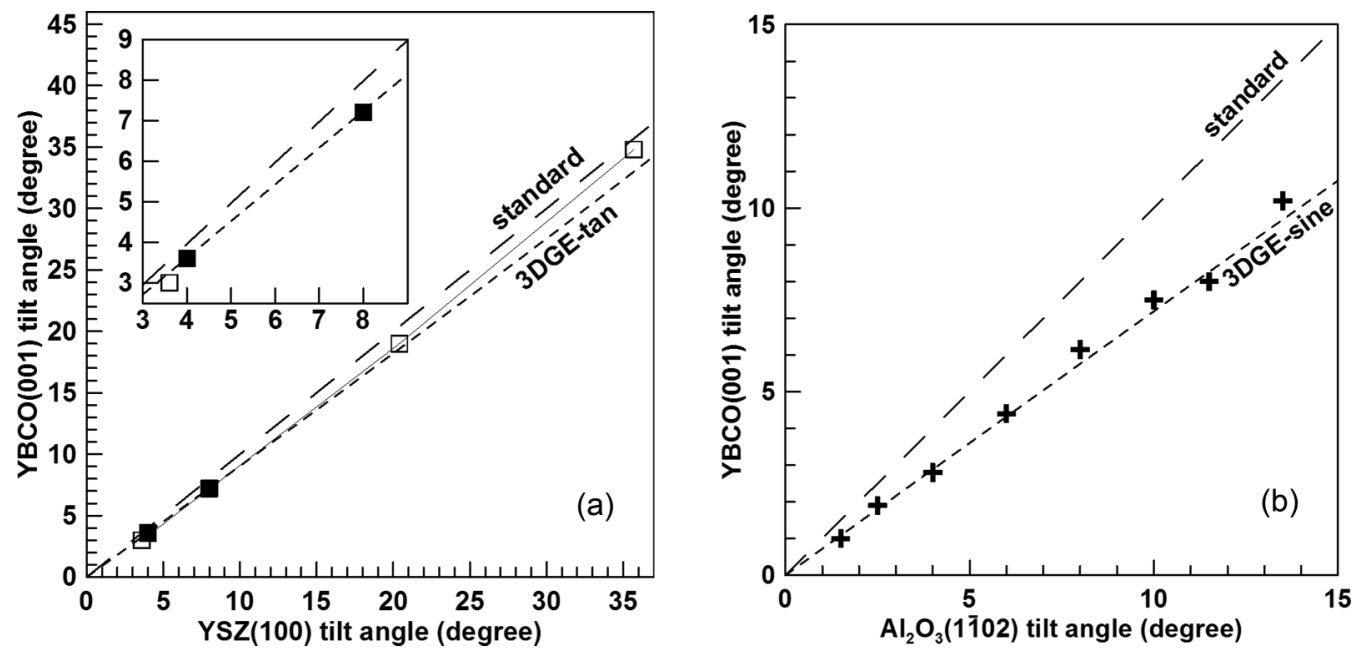

FIG. 14. Examples of 3DGE growth in studies of other groups. (a) YBCO deposition over YSZ TAS with (open squares, [23]) and without (solid squares, [22]) $\mathrm{Y}_{2} \mathrm{O}_{3}$ buffer layer. (b) $\mathrm{YBCO}$ deposition over $\mathrm{CeO}_{2}$ layer on sapphire TAS [22]. Note that $\mathrm{Y}_{2} \mathrm{O}_{3}$ over $\mathrm{YSZ}_{\text {and }} \mathrm{CeO} \mathrm{O}_{2}$ over sapphire grow in the standard mode.

1129 with roughness $R_{a}$ less than $2 \AA$, with no oriented or elongated 1130 features even for the TAS with high tilt angle. Observation of 1131 such a morphology implies the presence of damaged "amor1132 phous" areas at least on some part of the substrate surface. Ap1133 plicability of the geometrical growth mechanisms [Figs. 1(b) 1134 and 1(c)] was under serious doubts. Still the 3DGE growth 1135 mechanism was reliably detected for the majority of the 1136 tested top layer/bottom layer combinations. We may conclude, 1137 that even substrates that were not undergoing the surface 1138 reconstruction procedure provide good enough conditions for 1139 realization of the 3DGE growth. In fact, the 3DGE mechanism 1140 was not observed only when the bonds between film and 1141 bottom layer were broken by intense chemical interaction ${ }_{1142}$ (YBCO on YSZ, and, for some deposition conditions, on ${ }_{1143} \mathrm{CeO}_{2}$ and $\mathrm{BZO}$ ), and when the film and substrate were of the 1144 same crystal structure and the lattice mismatch between them 1145 was small $\sim 1 \%$ (YBCO on NGO).

${ }_{1146}$ Considering the effect of the surface morphology on the 1147 formation of the 3DGE films, we notice that even after severe 1148 step bunching ( $5^{\circ}$ tilted from $(0001)$ plane sapphire substrates 1149 after annealing at $1500{ }^{\circ} \mathrm{C}$ showed steps $\sim 40 \AA$ high [15]) 1150 the substrates provided good enough conditions for the 3DGE 1151 growth, i.e., both damaged amorphous surfaces and high steps 1152 after step bunching still allow growth by the 3DGE mode, 1153 implying a very high tolerance of this growth mode to the 1154 surface conditions.

1155 One more important issue referring to the substrate surface 1156 preparation is the orientation of the tilt axis. In our experi1157 ments the tilt axis was quite close to the [001] axis of the sub1158 strate, providing good conditions for "initial" matching of the 1159 film and substrate lattices. We cannot confidently claim that 1160 the film orientation will follow the 3DGE growth mode if the 1161 tilt axis is chosen along some other crystallographic direction. 1162 It is known that the change of the orientation of the surface 1163 features can influence orientation of the growing films (see, 1164 e.g., [32-34]). Experiments in semiconductor heterostructures 1165 showed a broad variety of effects of changing the tilt axis 1166 direction in the habit plane. Already in [8] the 3DGE growth 1167 mode of $\mathrm{Ga}(\mathrm{In})$ As was observed along the $\langle-110\rangle$ direction on the GaAs (001) plane, but not along orthogonal $\langle 110\rangle{ }_{1168}$ direction. In [11] $\mathrm{CdS}_{2}$ growth on sapphire showed 3DGE 1169 growth for all orientations of the tilt axis, while Si on sapphire, 1170 similarly to [8], showed the 3DGE behavior for tilt along only 1171 one of two orthogonal directions. The reason was different 1172 surface morphology resulting from miscut in nonsymmet- 1173 ric crystallographic directions [11]. The most complicated 1174 film tilt mechanism was observed in [35], when misfit level 1175 changed not only the film tilt angle, but also direction of tilt 1176 as seen from the habit plane. Ni grains orientations in [17] 1177 were "randomly" distributed along a predominant orientation, 1178 so the tilt axes on different grains were randomly oriented 1179 in the habit plane. All $\mathrm{CeO}_{2}$ grains showed the same 3DGE ${ }_{1180}$ growth mode, implying completely isotropic behavior of ceria ${ }_{1181}$ on $\mathrm{Ni}$ [17]. In our case changing the tilt axis to [111] $\left(45^{\circ}\right.$ tilt 1182 from the [001] direction in the (110) habit plane of NGO) 1183 may result in $3 \mathrm{DGE}$ growth with the same relations along 1184 the habit plane, or $45^{\circ}$ tilt of the film axes in habit plane, ${ }_{1185}$ or in $45^{\circ}$ tilt of the film axes normal to the habit plane-all ${ }_{1186}$ these initial orientational relations are possible and should be ${ }_{1187}$ checked experimentally for each pair top layer/bottom layer. ${ }_{1188}$ We even cannot claim that the 3DGE mechanism would be ${ }_{1189}$ preserved if the tilt axis is changed.

\section{E. Evidences of 3DGE growth in all-metaloxide heterostructures in the literature}

In the Introduction part of this paper we mentioned some ${ }_{1193}$ observations of the 3DGE growth mode by other groups. In 1194 fact, these observations are more numerous, but sometimes 1195 these data were misinterpreted or left without explanation. $\quad{ }_{1196}$

In [22] two combinations film-substrate (YBCO/YSZ and ${ }_{1197}$ $\mathrm{YBCO} / \mathrm{CeO}_{2} / \mathrm{Al}_{2} \mathrm{O}_{3}$ ) were studied in a wide angular range, 1198 $0^{\circ}-14^{\circ}$, orientation of both is finely described by the 3DGE ${ }_{1199}$ growth mechanism. YBCO grows over YSZ in the same 1200 way as $\mathrm{YBCO}$ over $\mathrm{CeO}_{2}$ in our experiments, with for- 1201 mation of $\mathrm{Ba}(\mathrm{Zr}, \mathrm{Y}) \mathrm{O}_{3}$ interlayer aligned with the YSZ 1202 SICPs [Fig. 14(a), solid squares]. YBCO over $\mathrm{CeO}_{2}$ fol- 1203 lows the 3DGE-sine growth mode assuming no chemical 1204 
1205 interaction between $\mathrm{YBCO}$ and $\mathrm{CeO}_{2}$, but $\mathrm{CeO}_{2}$ grows on 1206 sapphire according to the standard mode, with parallel SICPs : 1207 (001) $\mathrm{CeO}_{2} \|(1-102) \mathrm{Al}_{2} \mathrm{O}_{3}$ [Fig. 14(b)]. Actually, agree1208 ment with the 3DGE-tangent mode is marginally better, but 1209 we decided to keep the sine model in agreement with our 1210 results. Anyway, the difference between the sine and tangent 1211 modes in the $0^{\circ}-14^{\circ}$ range is less than $0.15^{\circ}$, being hardly 1212 distinguishable without special precautions during measure1213 ment. Note that a very good agreement is obtained using 1214 lattice constants for YBCO (11.685 $)$ ), YSZ (5.149 ̊), $\mathrm{CeO}_{2}$ $1215(5.405 \AA)$, and $\mathrm{Ba}(\mathrm{Y}, \mathrm{Zr}) \mathrm{O}_{3}(4.31 \AA)$, as measured in our 1216 experiments. No attempts to improve conformity by fitting the 1217 lattice constants was done.

1218 Another example of 3DGE growth is presented in [23]. 1219 The YBCO films buffered by a $\mathrm{Y}_{2} \mathrm{O}_{3}$ layer on a YSZ TAS 1220 showed a pronounced tilt of the SICP from the habit plane 1221 of the substrate in the range $3^{\circ}-36^{\circ}$. Again we get a good 1222 agreement between the presented numbers and calculated us1223 ing formula (1), assuming chemical interaction with formation 1224 of $\mathrm{Ba}(\mathrm{Y}, \mathrm{Zr}) \mathrm{O}_{3}$ with the same orientation of the SICP as that 1225 of the substrate and buffer layer [Fig. 14(a), open squares]. 1226 A tendency towards standard growth mode is observed at 1227 high angles, similarly to our results with BZO deposition on 1228 NGO TAS (Fig. 7), so we used the tangent model for the 1229 calculation. The agreement with the geometrical model is less 1230 accurate compared to [22] results, either due to an unknown 1231 composition of the product of chemical interaction in the 1232 beginning of the YBCO film growth, or simply as a result of 1233 the approximate numbers given in [23].

1234 Deposition of YBCO on YSZ layer in our experiments al1235 ways resulted in $c$-oriented YBCO films, in contradiction with 1236 the results [22], where 3DGE growth started after initial chem1237 ical interaction with formation of $\mathrm{Ba}(\mathrm{Y}, \mathrm{Zr}) \mathrm{O}_{3}$ layer. Yttrium 1238 segregation on the YSZ substrate surface during substrate 1239 preparation may be the reason, forming a $\mathrm{Y}(\mathrm{Zr})_{2} \mathrm{O}_{3} / \mathrm{YSZ}$ 1240 surface layer similar to $\mathrm{Y}_{2} \mathrm{O}_{3} / \mathrm{YSZ}$ in [23]. Such segregation 1241 is expected at high oxygen partial pressure, during annealing 1242 of the substrates after CMP, or during the prebake step imme1243 diately before deposition [36].

1244 Theis and Schlom [24] present a much more complicated 1245 heterostructure, where tetragonal $\mathrm{PbTiO}_{3}$ grows on a $\mathrm{SrTiO}_{3}$ 1246 TAS in two different orientations. Accurate modeling of the 1247 growth mode demands precise measurement of the lattice 1248 constants that are affected by stoichiometry and substrate1249 induced strain. Still the agreement with the geometrical model 1250 is more than qualitative. In [37] a significant distortion of the 1251 lattice should be taken into account to obtain agreement be1252 tween the geometrical model and the observed data. Reference 1253 [35] also notes importance of possible tetragonal distortions 1254 introduced by the substrate. These experiments demonstrate 1255 that the good agreement between calculated and measured 1256 values in our experiments is, in some sense, a coincidence, 1257 at least when such materials as $\mathrm{CeO}_{2}$ are considered. The 1258 structure of $\mathrm{CeO}_{2}$ is easily distorted by the substrate-induced 1259 strain, and the lattice constant of ceria strongly depends on 1260 density of oxygen vacancies generated during deposition (see, 1261 e.g., $[17,31,38])$. Reasonable agreement of the experimental 1262 data with the simplified model (1) is, to some extent, a for1263 tunate combination of circumstances, including oxygenation 1264 during and after deposition, and thickness of the films high enough to ignore the substrate-induced strain in the interface 1265 area.

The possible effect of lattice distortion can be illustrated ${ }_{1267}$ with results of [18] [see Fig. 2(a)]. The critical angle $\gamma_{c},{ }_{1268}$ calculated using standard ceria lattice constant, is smaller than 1269 the observed one $\left(30.4^{\circ}\right.$ and $32^{\circ}$, respectively). Taking into ac- 1270 count tetragonal distortion of ceria by the substrate-introduced 1271 tensile strain [assuming volume-preserving distortion and 1272 strain introduction only along the habit plane (110) NGO], 1273 we obtain $\gamma_{c}=31.2^{\circ}$, in better agreement with the measured ${ }_{1274}$ value. In fact, such estimation should consider also the strain 1275 introduced by the edges of the steps on the substrate surface, 1276 a decrease of the unit cell volume in the strained lattice, and ${ }_{1277}$ expansion of the lattice due to oxygen nonstoichiometry dur- 1278 ing deposition. All these factors are decreasing the calculated 1279 $\gamma_{c}$, so the accurate estimation would be in between $30.4^{\circ}{ }_{1280}$ and $31.2^{\circ}$. For our $\mathrm{CeO}_{2}$ films fabricated by PLD we do not ${ }_{1281}$ observe a substantial tetragonal distortion. The ceria lattice 1282 constant measured along the normal to habit plane (110) ${ }_{1283}$ NGO showed no dependence on angle and remained close to 1284 the value measured for the standard-oriented substrate. With 1285 increasing tilt angle the effect of mismatch with the habit ${ }_{1286}$ plane is decreasing, and the counteracting strain introduced by ${ }_{1287}$ the step edges is increasing. Constant lattice parameter, thus, 1288 means that the film grows independently on the substrate- 1289 induced strain and the lattice constant depends mainly on the 1290 deposition conditions (and corresponding density of oxygen 1291 vacancies in the film). An indirect proof of weak effect of 1292 tetragonal distortion for our films is a good match between 1293 the calculated and measured film tilt values (Fig. 4). 1294

Hoek et al. [39] provide another example of a more compli- 1295 cated mechanism matching top and bottom layers. The SICPs 1296 of $\mathrm{La}(\mathrm{Sr}) \mathrm{CuO}_{4}$ grown on a $26^{\circ}$ ramp etched of $\mathrm{Nd}(\mathrm{Ce}) \mathrm{CuO}_{4} \quad{ }_{1297}$ demonstrated a $3.3^{\circ}$ inclination to the SICPs of the bottom 1298 layer. The inclination monotonously changes with the tilt ${ }_{1299}$ angle of the ramp. The geometry of growth and the behavior 1300 of the inclination are similar to that discussed in our study, but 1301 application of the simple geometrical model gives a smaller 1302 tilt angle of $2.8^{\circ}$. Authors [39] suggest a more sophisticated ${ }_{1303}$ mechanism of matching of corresponding facets, say, (3 0 19), ${ }_{1304}$ of the top and bottom layers during growth, with simultaneous 1305 matching of both in-plane and out-of-plane lattice constants. 1306 This approach provides excellent agreement with the observed 1307 tilt angle between the SICPs of two layers. Similar results can 1308 be obtained for twin boundaries [39], where facet matching is 1309 the obvious mechanism of strain accommodation on the grain 1310 boundaries. References [11] and [40] also point out matching 1311 at higher symmetries (symmetric [11] and asymmetric [40] 1312 boundaries) as an alternative to the simple geometrical match- 1313 ing at the film-substrate interface.

\section{CONCLUSION}

We studied growth by PLD of metaloxide thin films on 1316 $\mathrm{NdGaO}_{3}$ substrates with the surface tilted from the stan- 1317 dard (110) crystallographic plane. Eight of ten studied top 1318 layer/bottom layer combinations showed a growth mode re- 1319 sulting in an inclination between the SICPs of the top layer 1320 and the corresponding SICPs of the bottom layer. The ob- 1321 served dependence of top layer tilt angle on the tilt angle of ${ }_{1322}$ 
1323 the bottom layer is well explained by a simple geometrical 1324 growth model, taking into account faceting of the surface 1325 of the bottom layer. The resulting growth mode depends 1326 both on standard atom-on-atom epitaxial matching along the 1327 tilt axis, graphoepitaxial matching in the normal to tilt axis 1328 direction in the substrate plane, and on the ratio of growth 1329 steps heights of the top and bottom layer, i.e., the matching 1330 of the top and the bottom layer is three dimensional. This 1331 growth mechanism may be described as a three-dimensional 1332 graphoepitaxial (3DGE) growth.

${ }_{1333}$ The 3DGE growth mechanism seems to be quite common 1334 for deposition on TAS with tilt angles more than $5^{\circ}$. PLD, 1335 rf sputtering [19,20], e-beam evaporation [18], and even LPE 1336 [15] provided conditions good enough for the 3DGE growth. 1337 No special substrate treatment is needed, even substrates with 1338 damaged amorphous surface or with step bunching [15] are 1339 suitable. The 3DGE growth mechanism is observed in mul1340 tilayer structures, both when the bottom layer follows 3DGE 1341 mode and when it grows with standard epitaxial relations. The 1342 3DGE growth was observed both with increase and decrease 1343 of the top layer tilt angle compared to the tilt angle of the 1344 bottom layer.

1345 Two different 3DGE dependencies may be distinguished 1346 in the high-angle range $\left(>15^{\circ}\right)$ : with a tendency towards 1347 standard growth above some threshold angle, and retaining 1348 3DGE behavior until a tilt angle of $45^{\circ}$ is reached, either 1349 by top or by bottom layer. The first type is better described 1350 by a tangent angular dependence, and usually is observed when a compressive strain is induced in the top layer. The ${ }_{1351}$ second type follows a sine dependence, and is usually seen 1352 for tensile-strained top layers. An increase over the calcu- 1353 lated value tilt is often observed in the range $5^{\circ}-10^{\circ}$ for the 1354 sine-type dependencies. In a simplified way the difference 1355 may be attributed to two different formation mechanisms, 1356 "overgrowth" and "simultaneous seeding." The first one forms 1357 the tilt different from the bottom layer when the growing 1358 grain overgrows another grain. For the second mechanism 1359 the top layer tilt is formed right when the grain is seeded. ${ }_{1360}$ Some material combinations showed both dependencies, for 1361 different deposition conditions. The reasons for realization of 1362 each of these dependencies should be clarified.

The results presented in this paper were obtained for ma- ${ }_{1364}$ terials with a simple cubic lattice (or the lattice that can be ${ }_{1365}$ reduced to a pseudocubic during growth at high deposition 1366 temperature). Reports from other groups point to the fact 1367 that a simple geometrical model of 3DGE growth may be of ${ }_{1368}$ limited validity in cases of more complex lattices, when the ${ }_{1369}$ top layer experiences significant distortions due to a bottom 1370 layer induced strain, or when the boundary between two layers 1371 exhibits mirror or central symmetry.

\section{ACKNOWLEDGMENTS}

The work was supported by Program of FASO of Russia. ${ }_{1374}$ I.K.B. also wishes to acknowledge FCT for its financial 1375 support (Grant No. IF/00582/2015).
[1] C. Tegenkamp, J. Phys.: Condens. Matter 21, 013002 (2009).

[2] M. Mukaida, Sh. Miyazawa, and M. Sasaura, Jpn. J. Appl. Phys. 30, L1474 (1991).

[3] J. Brotz, H. Fuess, T. Haage, J. Zegenhagen, Ch. Jooss, A. Forkl, and R. Warthmann, J. Appl. Phys. 85, 635 (1999).

[4] H. Song and T. S. Sudarshan, J. Cryst. Growth 371, 94 (2013).

[5] J. Zegenhagen, T. Haage, and Q. D. Jiang, Appl. Phys. A 67, 711 (1998).

[6] Ch. Chen, Zh. Chen, J. Zhang, and X.-J. Du, Sci. China Phys. Mech. Astron. 55, 2042 (2012).

[7] O. Igarashi, J. Appl. Phys. 42, 4035 (1971).

[8] H. Nagai, J. Appl. Phys. 45, 3789 (1974).

[9] G. H. Olsen and R. T. Smith, Phys. Status Solidi A 31, 739 (1975).

[10] O. Igarashi, Jpn. J. Appl. Phys. 15, 1435 (1976).

[11] M. Aindow and R. C. Pond, Philos. Mag. A 63, 667 (1991).

[12] F. Riesz, J. Appl. Phys. 79, 4111 (1996).

[13] J. P. Hirth and R. C. Pond, Philos. Mag. 90, 3129 (2010).

[14] A. Petkova, J. Wollschlaeger, H.-L. Guenter, and M. Henzler, Surf. Sci. 542, 211 (2003).

[15] R. Bachelet, G. Nahelou, A. Boulle, R. Guinebretiere, and A. Dauger, Prog. Solid State Chem. 33, 327 (2005).

[16] I. K. Bdikin, J. E. Mozhaeva, P. B. Mozhaev, C. S. Jacobsen, J. Bindslev Hansen, A. L. Kholkin, V. A. Luzanov, and I. M. Kotelyanskii, report on MATERIAIS 2005: XII Portuguese Materials Society Meeting, III International Materials Symposium, Aveiro, Portugal, 2005 (unpublished).
[17] J. D. Budai, W. Yang, N. Tamura, J.-S. Chung, J. Z. Tischler, B. C. Larson, G. E. Ice, Ch. Park, and D. P. Norton, Nat. Mater. 2, 487 (2003).

[18] I. M. Kotelyanskii and V. A. Luzanov (private communication, 1996).

[19] I. K. Bdikin, P. B. Mozhaev, G. A. Ovsyannikov, F. V. Komissinskii, I. M. Kotelyanskii, and E. I. Raksha, Phys. Solid State 43, 1611 (2001).

[20] I. K. Bdikin, P. B. Mozhaev, G. A. Ovsyannikov, P. V. Komissinski, and I. M. Kotelyanskii, Physica C 377, 26 (2002).

[21] P. B. Mozhaev, J. E. Mozhaeva, I. K. Bdikin, I. M. Kotelyanskii, V. A. Lusanov, J. Bindslev Hansen, C. S. Jacobsen, and A. L. Kholkin, Physica C 434, 105 (2006).

[22] E. Stepantsov, M. Tarasov, A. Kalabukhov, L. Kuzmin, and T. Claeson, J. Appl. Phys. 96, 3357 (2004).

[23] C. H. Mueller, P. H. Holloway, J. D. Budai, F. A. Miranda, and K. B. Bhasin, J. Mater. Res. 10, 810 (1995).

[24] C. D. Theis and D. G. Schlom, J. Mater. Res. 12, 1297 (1997).

[25] S. Kim, Y. Kang, and S. Baik, Thin Solid Films 256, 240 (1995).

[26] P. B. Mozhaev, A. V. Khoryushin, J. E. Mozhaeva, J.-C. Grivel, J. Bindslev Hansen, and C. S. Jacobsen, J. Supercond. Novel Magnetism 30, 2401 (2017).

[27] P. B. Mozhaev, J. E. Mozhaeva, I. K. Bdikin, T. Donchev, E. Mateev, T. Nurgaliev, C. S. Jacobsen, J. Bindslev Hansen, S. A. Zhgoon, and A. E. Barinov, Proc. SPIE 5401, Micro- and Nanoelectronics 2003 (2004).

[28] J. A. Alarco, G. Brorsson, Z. G. Ivanov, P.-A. Nilsson, E. Olsson, and M. Lofgren, Appl. Phys. Lett. 61, 723 (1992). 
[29] J.-H. Kim, S. Oh, and D. Youm, Thin Solid Films 305, 304 (1997).

[30] E. I. Givargizov, Thin Solid Films 189, 389 (1990).

[31] F. Wu, A. Pavlovska, D. J. Smith, R. J. Culbertson, B. J. Wilkens, and E. Bauer, Thin Solid Films 516, 4908 (2008).

[32] G. Brorsson, E. Olsson, Z. G. Ivanov, E. A. Stepantsov, J. A. Alarco, Yu. Boikov, T. Claeson, P. Berastegui, V. Langer, and M. Loefgren, J. Appl. Phys. 75, 7958 (1994).

[33] D. Vassiloyannis and P. M. Pardalos, Physica C 468, 147 (2008).

[34] M. Sunder and P. D. Moran, J. Electron. Mater. 38, 1931 (2009).
[35] A. Pesek, K. Hinger, F. Riesz, and K. Lischka, Semicond. Sci. Technol. 6, 705 (1991).

[36] X.-G. Wang, Surf. Sci. 602, L5 (2008).

[37] D. A. Neumann, H. Zabel, and H. Morkoc, J. Appl. Phys. 64, 3024 (1988).

[38] D. P. Norton, C. Park, J. D. Budai, S. J. Pennycook, and C. Prouteau, Appl. Phys. Lett. 74, 2134 (1999).

[39] M. Hoek, F. Coneri, N. Poccia, X. Renshaw Wang, X. Ke, G. Van Tendeloo, and H. Hilgenkamp, Appl. Phys. Lett. Mater. 3, 086101 (2015).

[40] B. W. Dodson, D. R. Myers, A. K. Datye, V. S. Kaushik, D. L. Kendall, and B. Martinez-Tovar, Phys. Rev. Lett. 61, 2681 (1988). 